\title{
WEYL STRUCTURES FOR PARABOLIC GEOMETRIES
}

\author{
ANDREAS ČAP and JAN SLOVÁK
}

\begin{abstract}
Motivated by the rich geometry of conformal Riemannian manifolds and by the recent development of geometries modeled on homogeneous spaces $G / P$ with $G$ semisimple and $P$ parabolic, Weyl structures and preferred connections are introduced in this general framework. In particular, we extend the notions of scales, closed and exact Weyl connections, and Rho-tensors, we characterize the classes of such objects, and we use the results to give a new description of the Cartan bundles and connections for all parabolic geometries.
\end{abstract}

\section{Introduction}

Cartan's generalized spaces are curved analogs of the homogeneous spaces $G / P$ defined by means of an absolute parallelism on a principal $P$-bundle. This very general framework was originally built in connection with the equivalence problem and Cartan's general method for its solution, cf. e.g. [11]. Later on, however, these ideas got much more attention. In particular, several well known geometries were shown to allow a canonical object of that type with suitable choice of semisimple $G$ and parabolic $P$, see e.g. [21]. Cartan's original approach was generalized and extended for all such groups, cf. [31], [25], [35], [8], and links to other areas were discovered, see e.g. [4], [3], [12]. The best known examples are the conformal Riemannian, projective, almost quaternionic, and CR structures and the common name adopted is parabolic geometries.

The relation to twistor theory renewed the interest in a good calculus for such geometries, which had to improve the techniques in conformal geometry and to extend them to other geometries. Many steps in this direction were done, see for example [32], [33], [34], [16] for classical methods in conformal geometry, and [2], [1], [15], [17], [18] for generalizations.

A new approach to this topic, motivated mainly by [26], [3], [4], was started in [9], [10]. The novelty consists in the combination of Lie algebraic tools with the frame bundle approach to all objects and we continue in this spirit here.

Received June 1, 2001. 
Our general setting for Weyl structures and scales has been also inspired by [1], [14].

In Section 2 we first outline some general aspects of parabolic geometries and then we present the basic objects like tangent and cotangent bundles and the curvature of the geometry in a somewhat new perspective. This will pave our way to the Weyl structures in the rest of the paper. Our basic references for Section 2 are [8] and [29], the reader may also consult [10]. For the classical point of view of over-determined systems, we refer to [31], [35] and the references therein.

The Weyl structures are introduced in the beginning of Section 3. Exactly as in the conformal Riemannian case, the class of Weyl structures underlying a parabolic geometry on a manifold $M$ is always an affine space modeled on oneforms on $M$ and each of them determines a linear connection on $M$. Moreover, the difference between the linear connection induced by a Weyl structure and the canonical Cartan connection is encoded in the so called Rho-tensor (used heavily in conformal geometry since the beginning of the century). Next, we define the bundles of scales as certain affine line bundles generalizing the distinguished bundles of conformal metrics, and we describe the correspondence between connections on these line bundles and the Weyl structures, see Theorem 3.8. On the way, we achieve explicit formulae for the deformation of Weyl structures and the related objects in Proposition 3.4, which offers a generalization for the basic ingredients of various calculi. The exact Weyl geometries are given by scales, i.e. by (global) sections of the bundles of scales, thus generalizing the class of Levi-Civita connections for conformal geometries. At the same time, this point of view leads to a new presentation of the canonical Cartan bundle as the bundle of connections on the bundle of scales (pulled back to the defining infinitesimal flag structure, cf. 2.6 and 3.8). In the end of Section 3, we define another class of distinguished local Weyl structures which achieve the best possible approximation of the canonical Cartan connections, see Theorem 3.5. In the conformal case, these normal Weyl structures improve the construction of the Graham's normal coordinates, cf. [24].

The last section is devoted to characterizations of all the objects related to a choice of a Weyl structure. More explicitly, the ultimate goal is to give a recipe how to decide which soldering forms and linear connections on a manifold $M$ equipped with a regular infinitesimal flag structure are obtained from a Weyl-structure and to compute the corresponding Rho-tensor. For this purpose, we define the general Weyl forms and their Weyl curvatures and the main step towards our aim is achieved in Theorem 4.1. Next, we introduce the total curvature of a Weyl form which is easier to interpret on the underlying manifold than the Weyl curvature. The characterization is then obtained by carefully analyzing the relation between these two curvatures. 
This entire paper focuses on the introduction of new structures and their nice properties. We should like to mention that essential use of these new concepts has appeared already in [10] and [5].

ACKNOWLEDGEMENTS. The initial ideas for this research evolved during the stay of the second author at the University of Adelaide in 1997, supported by the Australian Research Council. The final work and writing was done at the Erwin Schrödinger Institute for Mathematical Physics in Vienna. The second author also acknowledges the support from GACR, Grant Nr. 201/99/0296. Our thanks are also due to our colleagues for many discussions.

\section{Some background on parabolic geometries}

\section{1. $|k|$-graded Lie algebras}

Let $G$ be a real or complex semisimple Lie group, whose Lie algebra $\mathfrak{g}$ is equipped with a grading of the form

$$
\mathfrak{g}=\mathfrak{g}_{-k} \oplus \cdots \oplus \mathfrak{g}_{0} \oplus \cdots \oplus \mathfrak{g}_{k} .
$$

Such algebras $g$ are called $|k|$-graded Lie algebras.

Throughout this paper we shall further assume that no simple ideal of $\mathfrak{g}$ is contained in $\mathfrak{g}_{0}$ and that the (nilpotent) subalgebra $\mathfrak{g}_{-}=\mathfrak{g}_{-k} \oplus \cdots \oplus \mathfrak{g}_{-1}$ is generated by $\mathfrak{g}_{-1}$. Such algebras are sometimes called effective semisimple graded Lie algebras of $k$-th type, cf. [19], [31]. By $\mathfrak{p}_{+}$we denote the subalgebra $\mathfrak{g}_{1} \oplus \cdots \oplus \mathfrak{g}_{k}$ and by $\mathfrak{p}$ the subalgebra $\mathfrak{g}_{0} \oplus \mathfrak{p}_{+}$. We also write $\mathfrak{g}_{-}=\mathfrak{g}_{-k} \oplus$ $\cdots \oplus \mathfrak{g}_{-1}$, and $\mathfrak{g}^{j}=\mathfrak{g}_{j} \oplus \cdots \oplus \mathfrak{g}_{k}, j=-k, \ldots, k$.

It is well known that then $\mathfrak{p}$ is a parabolic subalgebra of $\mathfrak{g}$, and actually the filtration $\mathfrak{g}^{j}$ is completely determined by this subalgebra, see e.g. [35], Section 3. Thus all complex simple $|k|$-graded $\mathfrak{g}$ are classified by subsets of simple roots of complex simple Lie algebras (i.e. arbitrary placement of crosses over the Dynkin diagrams in the notation of [4]), up to conjugation. The real $|k|$ graded simple Lie algebras are classified easily by means of Satake diagrams: the $|k|$-grading of the complex simple $g$ induces a $|k|$-grading on a real form if and only if (i) only 'white' nodes in the Satake diagram have been crossed out, and, (ii) if a node is crossed out, then all nodes connected to this one by the double arrows in the Satake diagram have to be crossed out too, see [19] or [35] for more details. Very helpful notational conventions and computational recipes can be found in [4].

\section{2 .}

Let us recall basic properties of Lie groups $G$ with (effective) $|k|$-graded Lie algebras $\mathfrak{g}$. First of all, there is always a unique element $E \in \mathfrak{g}_{0}$ with the 
property $[E, Y]=j Y$ for all $Y \in \mathfrak{g}_{j}, j=-k, \ldots, k$, the grading element. Of course, $E$ belongs to the center $z$ of the reductive part $\mathfrak{g}_{0}$ of $\mathfrak{p} \subset \mathfrak{g}$.

The Killing form provides isomorphisms $\mathfrak{g}_{i}^{*} \simeq \mathfrak{g}_{-i}$ for all $i=-k, \ldots, k$ and, in particular, its restrictions to the center $z$ and the semisimple part $\mathfrak{g}_{0}^{s s}$ of $\mathfrak{g}_{0}$ are non-degenerate.

Now, there is the closed subgroup $P \subset G$ of all elements whose adjoint actions leave the $\mathfrak{p}$-submodules $\mathfrak{g}^{j}=\mathfrak{g}_{j} \oplus \cdots \oplus \mathfrak{g}_{k}$ invariant, $j=-k, \ldots, k$. The Lie algebra of $P$ is just $\mathfrak{p}$ and there is the subgroup $G_{0} \subset P$ of elements whose adjoint action leaves invariant the grading by $\mathrm{g}_{0}$-modules $\mathrm{g}_{i}, i=-k, \ldots, k$. This is the reductive part of the parabolic Lie subgroup $P$, with Lie algebra $\mathrm{g}_{0}$. We also define subgroups $P_{+}^{j}=\exp \left(\mathfrak{g}_{j} \oplus \cdots \oplus \mathrm{g}_{k}\right), j=1, \ldots, k$, and we write $P_{+}$instead of $P_{+}^{1}$. Obviously $P / P_{+}=G_{0}$ and $P_{+}$is nilpotent. Thus $P$ is the semidirect product of $G_{0}$ and the nilpotent part $P_{+}$. More explicitly, we have (cf. [8], Proposition 2.10, or [31], [35])

Proposition 2.1. For each element $g \in P$, there exist unique elements $g_{0} \in G_{0}$ and $Z_{i} \in \mathfrak{g}_{i}, i=1, \ldots, k$, such that

$$
g=g_{0} \exp Z_{1} \exp Z_{2} \ldots \exp Z_{k} .
$$

\subsection{Parabolic geometries}

Following Elie Cartan's idea of generalized spaces (see [28] for a recent reading), a curved analog of the homogeneous space $G / P$ is a right invariant absolute parallelism $\omega$ on a principal $P$-bundle $\mathscr{G}$ which reproduces the fundamental vector fields. In our approach, a (real) parabolic geometry $(\mathscr{G}, \omega)$ of type $G / P$ is a principal fiber bundle $\mathscr{G}$ with structure group $P$, equipped with a smooth one-form $\omega \in \Omega^{1}(\mathscr{G}, \mathfrak{g})$ satisfying

(1) $\omega\left(\zeta_{Z}\right)(u)=Z$ for all $u \in \mathscr{G}$ and fundamental fields $\zeta_{Z}, Z \in \mathfrak{p}$

(2) $\left(r^{b}\right)^{*} \omega=\operatorname{Ad}\left(b^{-1}\right) \circ \omega$ for all $b \in P$

(3) $\left.\omega\right|_{T_{u} \mathscr{G}}: T_{u} \mathscr{G} \rightarrow \mathfrak{g}$ is a linear isomorphism for all $u \in \mathscr{G}$.

In particular, each $X \in \mathfrak{g}$ defines the constant vector field $\omega^{-1}(X)$ defined by $\omega\left(\omega^{-1}(X)(u)\right)=X, u \in \mathscr{G}$. In this paper, we shall deal with smooth real parabolic geometries only. The one forms with properties (1)-(3) are called Cartan connections, cf. [28].

The morphisms between parabolic geometries $(\mathscr{G}, \omega)$ and $\left(\mathscr{G}^{\prime}, \omega^{\prime}\right)$ are principal fiber bundle morphisms $\varphi$ which preserve the Cartan connections, i.e. $\varphi: \mathscr{G} \rightarrow \mathscr{G}^{\prime}$ and $\varphi^{*} \omega^{\prime}=\omega$. 


\subsection{The curvature}

The structure equations define the horizontal smooth form $K \in \Omega^{2}(\mathscr{G}, \mathfrak{g})$ called the curvature of the Cartan connection $\omega$ :

$$
d \omega+\frac{1}{2}[\omega, \omega]=K .
$$

The curvature function $\kappa: \mathscr{G} \rightarrow \wedge^{2} \mathrm{~g}_{-}^{*} \otimes \mathfrak{g}$ is then defined by means of the parallelism

$$
\begin{aligned}
\kappa(u)(X, Y) & =K\left(\omega^{-1}(X)(u), \omega^{-1}(Y)(u)\right) \\
& =[X, Y]-\omega\left(\left[\omega^{-1}(X), \omega^{-1}(Y)\right]\right) .
\end{aligned}
$$

In particular, the curvature function is valued in the cochains for the second cohomology $H^{2}\left(\mathfrak{g}_{-}, \mathfrak{g}\right)$. Moreover, there are two ways how to split $\kappa$. We may consider the target components $\kappa_{i}$ according to the values in $\mathfrak{g}_{i}$. The whole $\mathfrak{g}_{-}$-component $\kappa_{-}$is called the torsion of the Cartan connection $\omega$. The other possibility is to consider the homogeneity of the bilinear maps $\kappa(u)$, i.e.

$$
\kappa=\sum_{\ell=-k+2}^{3 k} \kappa^{(\ell)}, \quad \kappa^{(\ell)}: \mathfrak{g}_{i} \times \mathfrak{g}_{j} \rightarrow \mathfrak{g}_{i+j+\ell} .
$$

Since we deal with semisimple algebras only, there is the codifferential $\partial^{*}$ which is adjoint to the Lie algebra cohomology differential $\partial$, see e.g. [23]. Consequently, there is the Hodge theory on the cochains which enables to deal very effectively with the curvatures. In particular, we may use several restrictions on the values of the curvature which turn out to be quite useful.

\subsection{Definition}

The parabolic geometry $(\mathscr{G}, \omega)$ with the curvature function $\kappa$ is called flat if $\kappa=0$, torsion-free if $\kappa_{-}=0$, normal if $\partial^{*} \circ \kappa=0$, and regular if it is normal and $\kappa^{(j)}=0$ for all $j \leq 0$.

Obviously, the morphisms of parabolic geometries preserve the above types and so we obtain the corresponding full subcategories of regular, normal, torsion free, and flat parabolic geometries of a fixed type $G / P$.

\subsection{Flag structures}

The homogeneous models for parabolic geometries are the real generalized flag manifolds $G / P$. Curved parabolic geometries look like $G / P$ infinitesimally. Indeed, the filtration of $\mathfrak{g}$ by the $\mathfrak{p}$-submodules $\mathfrak{g}^{j}$ is transfered to the right invariant filtration $T^{j} \mathscr{G}$ on the tangent space $T \mathscr{G}$ by the parallelism $\omega$. The tangent projection $T p: T \mathscr{G} \rightarrow T M$ then provides the filtration $T M=$ 
$T^{-k} M \supset T^{-k+1} M \supset \cdots \supset T^{-1} M$ of the tangent space of the underlying manifold $M$. Moreover, the structure group of the associated graded tangent space Gr $T M=\left(T^{-k} M / T^{-k+1} M\right) \oplus \cdots \oplus\left(T^{-2} M / T^{-1} M\right) \oplus T^{-1} M$ reduces automatically to $G_{0}$ since $\mathscr{G}_{0}=\mathscr{G} / P_{+}$clearly plays the role of its frame bundle. The following lemma is not difficult to prove, see e.g. [27], Lemma 2.11.

Lemma. Let $(\mathscr{G}, \omega)$ be a parabolic geometry, $\kappa$ its curvature function. Then $\kappa^{(j)}=0$ for all $j<0$ if and only if the Lie bracket of vector fields on $M$ is compatible with the filtration, i.e. $[\xi, \eta]$ is a section of $T^{i+j} M$ for all sections $\xi$ of $T^{i} M$, and $\eta$ of $T^{j} M$. Hence it defines an algebraic bracket $\{,\}_{\text {Lie }}$ on Gr TM. Moreover, this bracket coincides with the algebraic bracket $\{,\}_{\mathfrak{g}_{0}}$ defined on $\mathrm{Gr} T M$ by means of the $G_{0}$-structure if and only if $\kappa^{(j)}=0$ for all $j \leq 0$.

We call the filtrations of $T M$ with reduction of $\operatorname{Gr} T M$ to $G_{0}$ satisfying the very last condition of the lemma the regular infinitesimal flag structures of type $\mathrm{g} / \mathfrak{p}$. In fact, the structures clearly depend on the choice of the Lie group $G$ with the given Lie algebra g. This choice is always encoded already in $G_{0}$. On the other hand, there are always several distinguished choices, e.g. the full automorphism group of $\mathfrak{g}$, the adjoint group, and the unique connected and simply connected group. In the conformal geometries these choices lead to conformal Riemannian manifolds, oriented conformal menifolds, and (oriented) conformal spin manifolds, respectively. Obviously, the various choices of $G$ do not matter much locally and we shall not discuss them explicitly in this paper.

The $G_{0}$ structures on Gr $T M$ are equivalent to the frame forms of length one defined and used in [8] while the condition $\kappa^{(j)}=0$ for all $j \leq 0$ is equivalent to the structure equations for these frame forms imposed in the construction of [8]. In view of this relation, we also call our bundles $\mathscr{G}_{0}$ equipped with the regular infinitesimal flag structures the $P$-frame bundles of degree one. In particular, we obtain (see [8], Section 3)

THEOREM 2.2. There is the bijective correspondence between the isomorphism classes of regular parabolic geometries of type $G / P$ and the regular infinitesimal flag structures of type $\mathfrak{g} / \mathfrak{p}$ on $M$, except for one series of one-graded, and one series of two-graded Lie algebras $\mathrm{g}$ for which $\mathrm{H}^{1}\left(\mathfrak{g}_{-}, \mathrm{g}\right)$ is nonzero in homogeneous degree one.

Both types of the exceptional geometries from the Theorem will be mentioned in the examples below. 


\subsection{Example}

The parabolic geometries with $|1|$-graded Lie algebras $g$ are called irreducible. Their tangent bundles do not carry any nontrivial natural filtration and this irreducibility of $T M$ is reflected in the name. The classification of all such simple real Lie algebras is well known (cf. [22] or 2.1 above). We may list all the corresponding geometries, up to the possible choices of the groups $G_{0}$, roughly as follows:

$A_{\ell}$ the split form, $\ell>2$ - the almost Grassmannian structures with homogeneous models of $p$-planes in $\mathrm{R}^{\ell+1}, p=1, \ldots, \ell$. The choice $p=1$ yields the projective structures which represent one of the two exceptions in Theorem 2.2.

$A_{\ell}$ the quaternionic form, $\ell=2 p+1>2-$ the almost quaternionic geometries in dimensions $4 p$, and more general geometries modeled on quaternionic Grassmannians.

$A_{\ell}$ one type of geometry for the algebra $\mathfrak{s} \mathfrak{H}(p, p), \ell=2 p-1$.

$B_{\ell}$ the (pseudo) conformal geometries in all odd dimensions $2 m+1 \geq 3$.

$C_{\ell}$ the split form, $\ell>2$ - the almost Lagrangian geometries modeled on the Grassmann manifold of maximal Lagrangian subspaces in the symplectic $\mathrm{R}^{2 \ell}$.

$C_{\ell}$ another type of geometry corresponding to the algebra $\mathfrak{s p}(p, p), \ell=2 p$.

$D_{\ell}$ the (pseudo) conformal geometries in all even dimensions $m \geq 4$.

$D_{\ell}$ the real almost spinorial geometries with $g=\mathfrak{s} \mathfrak{D}(p, 2 \ell-p), p=$ $1, \ldots, \ell-2$.

$D_{\ell}$ the quaternionic almost spinorial geometries with $\mathfrak{g}=\mathfrak{t}^{*}(\ell, \mathrm{H})$.

$E_{6}$ the split form $E I-$ exactly one type with $\mathfrak{g}_{0}=\mathfrak{s} \mathfrak{D}(5,5) \oplus \mathrm{R}$ and $\mathfrak{g}_{-1}=$ $\mathrm{R}^{16}$.

$E_{6}$ the real form $E I V$ - exactly one type with $\mathfrak{g}_{0}=\mathfrak{s} \mathfrak{D}(1,9) \oplus \mathrm{R}$ and $g_{-1}=\mathrm{R}^{16}$.

$E_{7}$ the split form $E V$ - exactly one type with $\mathfrak{g}_{0}=E I \oplus \mathrm{R}$ and $\mathfrak{g}_{-1}=\mathrm{R}^{27}$.

$E_{7}$ the real form $E V I I$ - exactly one type with $\mathfrak{g}_{0}=E I V \oplus \mathrm{R}$ and $\mathfrak{g}_{-1}=$ $\mathrm{R}^{27}$.

\subsection{Example}

The parabolic contact geometries form another important class. They correspond to $|2|$-graded Lie algebras $\mathfrak{g}$ with one-dimensional top components $\mathfrak{g}_{2}$. Thus the regular infinitesimal structures are equivalent to contact geometric 
structures, together with the reduction of the graded tangent space to the subgroup $G_{0}$ in the group of contact transformations. The only exceptions are the so called projective contact structures $\left(C_{\ell}\right.$ series of algebras) where more structure has to be added, see e.g. [8]. The general classification scheme allows a simple formulation for the contact cases: The dimension one condition on $g_{2}$ yields the prescription which simple roots have to be crossed while the prescribed length two of the grading gives further restrictions. The outcome may be expressed as (see [19], [35]):

PROPOSITION. Each non-compact real simple Lie algebra g admits a unique grading of contact type (up to conjugacy classes), except $\mathrm{g}$ is one of $\mathfrak{g}(2, \mathrm{R})$, $\mathfrak{S l}(\ell, \mathrm{H}), \mathfrak{s p}(p, q), \mathfrak{s} \mathfrak{D}(1, q), E I V, F I I$ and in these cases no such gradings exist.

The best known examples are the non-degenerate hypersurface type $C R$ geometries (with signature $(p, q)$ of the Levi form) which are exactly the torsion free regular parabolic geometries with $\mathfrak{g}=\mathfrak{g} \mathfrak{H}(p+1, q+1)$, see e.g. [8], Section 4.14-4.16. The real split forms of the same complex algebras give rise to the so called almost Lagrangian contact geometries, cf. [30].

\subsection{Example}

The previous two lists of geometries include those with most simple infinitesimal flag structures. The other extreme is provided by the real parabolic geometries with most complicated flags in each tangent space, i.e. those corresponding to the Borel subgroups $P \subset G$. Here we need to cross out all nodes in the Satake diagram and so there must not be any black ones. Thus all real split forms, $\mathfrak{H} \mathfrak{H}(p, p), \mathfrak{S} \mathfrak{D}(\ell-1, \ell+1)$, and EII list all real forms which admit the right grading.

\subsection{Natural bundles}

Consider a fixed parabolic geometry $(\mathscr{G}, \omega)$ over a manifold $M$. Then each $P$-module $\mathrm{V}$ defines the associated bundle $V M=\mathscr{G} \times{ }_{P} \mathrm{~V}$ over $M$. In fact, this is a functorial construction which may be restricted to all subcategories of parabolic geometries mentioned in 2.5.

Similarly, we may treat bundles associated to any action $P \rightarrow \operatorname{Diff}(\mathbf{S})$ on a manifold $\mathrm{S}$, the standard fiber for $S M=\mathscr{G} \times{ }_{P} \mathrm{~S}$. We shall meet only natural vector bundles defined by $P$-modules in this paper, however.

There is a special class of natural (vector) bundles defined by $G$-modules W. Such natural bundles are called tractor bundles, see [2], [7] for historical remarks. We shall denote them by the script letters here and often omit the base manifold $M$ from the notation. We may view each such tractor bundle $\mathscr{W} M$ as associated to the extended principal fiber bundle $\tilde{\mathscr{G}}=\mathscr{G} \times{ }_{P} G$, i.e. 
$\mathscr{W}=\tilde{\mathscr{G}} \times_{G} \mathrm{~W}$. Now, the Cartan connection $\omega$ on $\mathscr{G}$ extends uniquely to a principal connection form $\tilde{\omega}$ on $\tilde{\mathscr{G}}$, and so there is the induced linear connection on each such $\mathscr{W}$. With some more careful arguments, this construction may be extended to all $(\mathfrak{g}, P)$-modules $\mathrm{W}$, i.e. $P$-modules with a fixed extension of the induced representation of $\mathfrak{p}$ to a representation of $\mathfrak{g}$ compatible with the $P$-action, see [7], Section 2. One of the achievements of the latter paper is the equivalent treatment of the regular parabolic geometries entirely within the framework of the tractor bundles, inclusive the discussion of the canonical connections.

\subsection{Adjoint tractors}

It seems that the most important natural bundle is the adjoint tractor bundle $\mathscr{A}=\mathscr{G} \times{ }_{P} \mathrm{~g}$ with respect to the adjoint action Ad of $G$ on $\mathrm{g}$. The $P$-submodules $\mathfrak{g}^{j} \subset \mathfrak{g}$ give rise to the filtration

$$
\mathscr{A}=\mathscr{A}^{-k} \supset \mathscr{A}^{-k+1} \supset \cdots \supset \mathscr{A}^{0} \supset \mathscr{A}^{1} \supset \cdots \supset \mathscr{A}^{k}
$$

by the natural subbundles $\mathscr{A}^{j}=\mathscr{G} \times{ }_{P} \mathfrak{g}^{j}$. Moreover, the associated graded natural bundle (often denoted by the abuse of notation by the same symbol again)

$$
\operatorname{Gr} \mathscr{A}^{=} \mathscr{A}_{-k} \oplus \cdots \oplus \mathscr{A}_{-1} \oplus \mathscr{A}_{0} \oplus \mathscr{A}_{1} \oplus \cdots \oplus \mathscr{A}_{k}
$$

with $\mathscr{A}_{j}=\mathscr{A}^{j} / \mathscr{A}^{j+1}$ is available. By the very definition, there is the algebraic bracket on $\mathscr{A}$ defined by means of the Lie bracket in $\mathrm{g}$ (since the Lie bracket is Ad-equivariant), which shows up on the graded bundle as

$$
\{,\}: \mathscr{A}_{i} \times \mathscr{A}_{j} \rightarrow \mathscr{A}_{i+j} .
$$

For the same reason, the Killing form defines a pairing on Gr $\mathscr{A}$ such that $\mathscr{A}_{i}^{*}=\mathscr{A}_{-i}$, and the algebraic codifferential $\partial^{*}$, cf. 2.4, defines natural algebraic mappings

$$
\partial^{*}: \wedge^{k+1} \mathscr{A}^{1} \otimes \mathscr{A} \rightarrow \wedge^{k} \mathscr{A}^{1} \otimes \mathscr{A}
$$

which are homogeneous of degree zero with respect to the gradings in Gr $\mathscr{A}$.

Similarly to the notation for $\mathfrak{g}$, we also write $\mathscr{A}_{+}=\mathscr{A}^{1}, \mathscr{A}_{-}=\mathscr{A} / \mathscr{A}^{0}$ for bundles associated either to $\mathscr{G}$ or $\mathscr{G}_{0}$. Thus $\mathscr{A}=\mathscr{A}_{-}+\mathscr{A}_{0}+\mathscr{A}_{+}$, understood either as composition series induced by the filtration, or (for the assotiated grades) direct sum of invariant subbundles, respectively.

\subsection{Tangent and cotangent bundles}

For each parabolic geometry $(\mathscr{G}, \omega), p: \mathscr{G} \rightarrow M$, the absolute parallelism defines the identification

$$
\mathscr{G} \times{ }_{P}(\mathfrak{g} / \mathfrak{p}) \simeq T M, \quad \mathscr{G} \times \mathfrak{g}_{-} \ni(u, X) \mapsto T p\left(\omega^{-1}(X)(u)\right) .
$$


In other words, the tangent spaces $T M$ are natural bundles equipped with the filtrations which correspond to the Lie algebras $g_{-}$viewed as the $P$-modules $\mathfrak{g} / \mathfrak{p}$ with the induced Ad-actions. Equivalently, the tangent spaces are the quotients

$$
T M=\mathscr{A} / \mathscr{A}^{0}
$$

of the adjoint tractor bundles. Therefore, the induced graded tangent spaces Gr $T M$ are exactly the negative parts of the graded adjoint tractor bundles

$$
\operatorname{Gr} T M=\mathscr{A}_{-k} \oplus \cdots \oplus \mathscr{A}_{-1} .
$$

Moreover, the definition of the algebraic bracket on $\mathscr{A}$ implies immediately that the bracket induced by the Lie bracket of vector fields on Gr $T M$ for regular infinitesimal flag structures on $M$ coincides with $\{$,$\} .$

Now, the cotangent bundles clearly correspond to

$$
T^{*} M=\mathscr{G} \times_{P} \mathfrak{p}_{+} \simeq \mathscr{A}^{1}
$$

and so the graded cotangent space is identified with

$$
\operatorname{Gr} T^{*} M=\mathscr{A}_{1} \oplus \cdots \oplus \mathscr{A}_{k} .
$$

Finally, the pairing of a one-form and a vector field is given exactly by the canonical pairing of $\mathscr{A} / \mathscr{A}^{1}$ and $\mathscr{A}^{1}$ induced by the Killing form.

\subsection{3 .}

The first important observation about the adjoint tractors and their links to tangent and cotangent spaces is that the curvature $K$ of the parabolic geometry $(\mathscr{G}, \omega)$ is in fact a section of $\Lambda^{2}\left(\mathscr{A} / \mathscr{A}^{0}\right)^{*} \otimes \mathscr{A}$ whose frame form is the curvature function $\kappa$. Thus, the curvature is a two-form on the underlying manifold $M$ valued in the adjoint tractors and all the conditions on the curvature discussed in 2.5 are expressed by natural algebraic operations on the adjoint tractors.

The remarkable relation of both tangent and cotangent spaces to the positive and negative parts of the adjoint tractors is the most important tool in what follows. In particular, let us notice already here that once we are given a reduction of the structure group $P$ of $\mathscr{G}$ to its reductive part $G_{0}$, the adjoint tractor bundles are identified with their graded versions and both tangent and cotangent bundles are embedded inside of $\mathscr{A}$. 


\section{Weyl-structures}

\subsection{Definition}

Let $(p: \mathscr{G} \rightarrow M, \omega$ ) be a parabolic geometry on a smooth manifold $M$, and consider the underlying principal $G_{0}$-bundle $p_{0}: \mathscr{G}_{0} \rightarrow M$ and the canonical projection $\pi: \mathscr{G} \rightarrow \mathscr{G}_{0}$. A Weyl-structure for $(\mathscr{G}, \omega)$ is a global $G_{0}$-equivariant smooth section $\sigma: \mathscr{G}_{0} \rightarrow \mathscr{G}$ of $\pi$.

Proposition 3.1. For any parabolic geometry $(p: \mathscr{G} \rightarrow M, \omega)$, there exists a Weyl-structure. Moreover, if $\sigma$ and $\hat{\sigma}$ are two Weyl-structures, then there is a unique smooth section $\Upsilon=\left(\Upsilon_{1}, \ldots, \Upsilon_{k}\right)$ of $\mathscr{A}_{1} \oplus \cdots \oplus \mathscr{A}_{k}$ such that

$$
\hat{\sigma}(u)=\sigma(u) \exp \left(\Upsilon_{1}(u)\right) \ldots \exp \left(\Upsilon_{k}(u)\right) .
$$

Finally, each Weyl-structure $\sigma$ and section $\Upsilon$ define another Weyl-structure $\hat{\sigma}$ by the above formula.

Proof. We can choose a finite open covering $\left\{U_{1}, \ldots, U_{N}\right\}$ of $M$ such that both $\mathscr{G}$ and $\mathscr{G}_{0}$ are trivial over each $U_{i}$. Since by Proposition $2.1 P$ is the semidirect product of $G_{0}$ and $P_{+}$it follows immediately that there are smooth $G_{0}$-equivariant sections $\sigma_{i}: p_{0}^{-1}\left(U_{i}\right) \rightarrow p^{-1}\left(U_{i}\right)$. Moreover, we can find open subsets $V_{i}$ such that $\bar{V}_{i} \subset U_{i}$ and such that $\left\{V_{1}, \ldots, V_{N}\right\}$ still is a covering of $M$.

Now from Proposition 2.1 and the Baker-Campbell-Hausdorff formula it follows that there is a smooth mapping $\Psi: p_{0}^{-1}\left(U_{1} \cap U_{2}\right) \rightarrow \mathfrak{p}_{+}$such that $\sigma_{2}(u)=\sigma_{1}(u) \exp (\Psi(u))$. Equivariance of $\sigma_{1}$ and $\sigma_{2}$ immediately implies that $\Psi(u \cdot g)=\operatorname{Ad}\left(g^{-1}\right)(\Psi(u))$ for all $g \in G_{0}$. Now let $f: M \rightarrow$ $[0,1]$ be a smooth function with support contained in $U_{2}$, which is identically one on $V_{2}$ and define $\sigma: p_{0}^{-1}\left(U_{1} \cup V_{2}\right) \rightarrow p^{-1}\left(U_{1} \cup V_{2}\right)$ by $\sigma(u)=$ $\sigma_{1}(u) \exp \left(f\left(p_{0}(u)\right) \Psi(u)\right)$ for $u \in U_{1}$ and by $\sigma(u)=\sigma_{2}(u)$ for $u \in V_{2}$. Then obviously these two definitions coincide on $U_{1} \cap V_{2}$, so $\sigma$ is smooth. Moreover, from the equivariance of the $\sigma_{i}$ and of $\Psi$ one immediately concludes that $\sigma$ is equivariant. Similarly, one extends the section next to $U_{1} \cup V_{2} \cup V_{3}$ and by induction one reaches a globally defined smooth equivariant section.

If $\hat{\sigma}$ and $\sigma$ are two global equivariant sections, then applying Proposition 2.1 directly, we see that there are smooth maps $\Upsilon_{i}: \mathscr{G}_{0} \rightarrow \mathrm{g}_{i}$ for $i=1, \ldots, k$ such that $\hat{\sigma}(u)=\sigma(u) \exp \left(\Upsilon_{1}(u)\right) \ldots \exp \left(\Upsilon_{k}(u)\right)$. As above, equivariance of $\hat{\sigma}$ and $\sigma$ implies that $\Upsilon_{i}(u \cdot g)=\operatorname{Ad}\left(g^{-1}\right)\left(\Upsilon_{i}(u)\right)$ for all $g \in G_{0}$. Hence, $\Upsilon_{i}$ corresponds to a smooth section of $\mathscr{A}_{i}$. The last statement of the Proposition is obvious now. 


\subsection{Weyl connections}

We can easily relate a Weyl-structure $\sigma: \mathscr{G}_{0} \rightarrow \mathscr{G}$ to objects defined on the manifold $M$ by considering the pullback $\sigma^{*} \omega$ of the Cartan connection $\omega$ along the section $\sigma$. Clearly, $\sigma^{*} \omega$ is a g-valued one-form on $\mathscr{G}_{0}$, which by construction is $G_{0}$-equivariant, i.e. $\left(r^{g}\right)^{*}\left(\sigma^{*} \omega\right)=\operatorname{Ad}\left(g^{-1}\right) \circ \sigma^{*} \omega$ for all $g \in G_{0}$. Since $\operatorname{Ad}\left(g^{-1}\right)$ preserves the grading of $\mathfrak{g}$, in fact each component $\sigma^{*} \omega_{i}$ of $\sigma^{*} \omega$ is a $G_{0}$-equivariant one form with values in $\mathfrak{g}_{i}$.

Now consider a vertical tangent vector on $\mathscr{G}_{0}$, i.e. the value $\zeta_{A}(u)$ of a fundamental vector field corresponding to some $A \in \mathfrak{g}_{0}$. Since $\sigma$ is $G_{0}$-equivariant, we conclude that $T_{u} \sigma \cdot \zeta_{A}(u)=\zeta_{A}(\sigma(u))$, where the second fundamental vector field is on $\mathscr{G}$. Consequently, we have $\sigma^{*} \omega\left(\zeta_{A}\right)=\omega\left(\zeta_{A}\right)=A \in \mathfrak{g}_{0}$. Thus, for $i \neq 0$ the form $\sigma^{*} \omega_{i}$ is horizontal, while $\sigma^{*} \omega_{0}$ reproduces the generators of fundamental vector fields.

From this observation, it follows immediately, that for $i \neq 0$, the form $\sigma^{*} \omega_{i}$ descends to a smooth one form on $M$ with values in $\mathscr{A}_{i}$, which we denote by the same symbol, while $\sigma^{*} \omega_{0}$ defines a principal connection on the bundle $\mathscr{G}_{0}$. This connection is called the Weyl connection of the Weyl structure $\sigma$.

\subsection{Soldering forms and Rho-tensors}

We view the positive components of $\sigma^{*} \omega$ as a one-form

$$
\mathrm{P}=\sigma^{*}\left(\omega_{+}\right) \in \Omega^{1}\left(M ; \mathscr{A}_{1} \oplus \cdots \oplus \mathscr{A}_{k}\right)
$$

with values in the bundle $\mathscr{A}_{1} \oplus \cdots \oplus \mathscr{A}_{k}$. We call it the Rho-tensor of the Weylstructure $\sigma$. This is a generalization of the tensor $\mathbf{P}_{a b}$ well known in conformal geometry.

Since $\omega$ restricts to a linear isomorphism in each tangent space of $\mathscr{G}$, we see that the form

$$
\sigma^{*} \omega_{-}=\left(\sigma^{*} \omega_{-k}, \ldots, \sigma^{*} \omega_{-1}\right) \in \Omega^{1}\left(M, \mathscr{A}_{-k} \oplus \cdots \oplus \mathscr{A}_{-1}\right)
$$

induces an isomorphism

$$
T M \cong \mathscr{A}_{-k} \oplus \cdots \oplus \mathscr{A}_{-1} \cong \mathrm{Gr} T M .
$$

We will denote this isomorphism by

$$
\xi \mapsto\left(\xi_{-k}, \ldots, \xi_{-1}\right) \in \mathscr{A}_{-k} \oplus \cdots \oplus \mathscr{A}_{-1}
$$

for $\xi \in T M$. In particular, each fixed $u \in \mathscr{G}_{0}$ provides the identification of $T_{p_{0}(u)} M \cong \mathfrak{g}_{-}$compatible with the grading. Thus, the choice of a Weyl structure $\sigma$ provides a reduction of the structure group of $T M$ to $G_{0}$ (by means of the soldering form $\sigma^{*} \omega_{-}$on $\mathscr{G}_{0}$ ), the linear connection on $M$ (the Weyl connection $\left.\sigma^{*} \omega_{0}\right)$, and the Rho-tensor P. 


\subsection{Remarks}

As discussed in 2.6 above, there is the underlying frame form of length one on $\mathscr{G}_{0}$ which is the basic structure from which the whole parabolic geometry $(\mathscr{G}, \omega)$ may be reconstructed, with exceptions mentioned explicitly in 2.7 and 2.8. By definition, for $i<0$ and $\xi \in T^{i} \mathscr{G}_{0}$ this frame form can be computed by choosing any lift of $\xi$ to a tangent vector on $\mathscr{G}$ and then taking the $\mathfrak{g}_{i}$ component of the value of $\omega$ on this lift. In particular, we can use $T \sigma \cdot \xi$ as the lift, which implies that the restriction of $\sigma^{*} \omega_{i}$ (viewed as a form on $\mathscr{G}_{0}$ ) to $T^{i} \mathscr{G}_{0}$ coincides with the $\mathfrak{g}_{i}$-component of the frame form of length one. This in turn implies that the restriction of $\sigma^{*} \omega_{i}$ (viewed as a form on $M$ ) to $T^{i} M$ coincides with the canonical projection $T^{i} M \rightarrow \mathscr{A}_{i}=T^{i} M / T^{i+1} M$.

There is also another interpretation of the objects on $M$ induced by the choice of a Weyl-structure that will be very useful in the sequel. Namely, consider the form

$$
\sigma^{*} \omega_{\leq 0}=\sigma^{*} \omega_{-k} \oplus \cdots \oplus \sigma^{*} \omega_{0} \in \Omega^{1}\left(\mathscr{G}_{0}, \mathfrak{g}_{-k} \oplus \cdots \oplus \mathfrak{g}_{0}\right) .
$$

We have seen above that this form is $G_{0}$-equivariant, it reproduces the generators of fundamental vector fields, and restricted to each tangent space, it is a linear isomorphism. Thus $\sigma^{*} \omega_{\leq 0}$ defines a Cartan connection on the principal $G_{0}$-bundle $p_{0}: \mathscr{G}_{0} \rightarrow M$. In the case of the irreducible parabolic geometries, these connections are classical affine connections on the tangent space $T M$ belonging to its reduced structure group $G_{0}$.

\subsection{Bundles of scales}

As we have seen in 3.2, 3.3 above, choosing a Weyl-structure $\sigma: \mathscr{G}_{0} \rightarrow \mathscr{G}$ leads to several objects on the manifold $M$. Now the next step is to show that in fact a small part of these data is sufficient to completely fix the Weyl-structure. More precisely, we shall see below that even the linear connections induced by the Weyl connection $\sigma^{*} \omega_{0}$ on certain oriented line bundles suffice to pin down the Weyl-structure. Equivalently, one can use the corresponding frame bundles, which are principal bundles with structure group $\mathrm{R}^{+}$. The principal bundles appropriate for this purpose are called bundles of scales.

To define these bundles, we have to make a few observations: A principal $\mathrm{R}^{+}$-bundle associated to $\mathscr{G}_{0}$ is determined by a homomorphism $\lambda: G_{0} \rightarrow \mathrm{R}^{+}$. The derivative of this homomorphism is a linear map $\lambda^{\prime}: \mathfrak{g}_{0} \rightarrow \mathbf{R}$. Now $\mathfrak{g}_{0}$ splits as the direct sum $z\left(\mathfrak{g}_{0}\right) \oplus \mathfrak{g}_{0}^{s s}$ of its center and its semisimple part, and $\lambda^{\prime}$ automatically vanishes on the semisimple part. Moreover, as discussed in 2.2 the restriction of the Killing form $B$ of $\mathfrak{g}$ to the subalgebra $\mathfrak{g}_{0}$ is non-degenerate, and one easily verifies that this restriction respects the above splitting. In particular, the restriction of $B$ to $z\left(\mathfrak{g}_{0}\right)$ is still non-degenerate and thus there is a unique element $E_{\lambda} \in \jmath\left(\mathfrak{g}_{0}\right)$ such that $\lambda^{\prime}(A)=B\left(E_{\lambda}, A\right)$ for all $A \in \mathfrak{g}_{0}$. 
Next, the action of the element $E_{\lambda} \in z\left(g_{0}\right)$ on any $G_{0}$-irreducible representation commutes with the action of $G_{0}$, and thus is given by a scalar multiple of the identity by Schur's lemma.

\section{Definition}

An element $E_{\lambda}$ of $\precsim\left(\mathfrak{g}_{0}\right)$ is called a scaling element if and only if $E_{\lambda}$ acts by a nonzero real scalar on each $G_{0}$-irreducible component of $\mathfrak{p}_{+}$. A bundle of scales is a principal $\mathrm{R}^{+}$bundle $\mathscr{L}^{\lambda} \rightarrow M$ which is associated to $\mathscr{G}_{0}$ via a homomorphism $\lambda: G_{0} \rightarrow \mathrm{R}^{+}$, whose derivative is given by $\lambda^{\prime}(A)=B\left(E_{\lambda}, A\right)$ for some scaling element $E_{\lambda} \in z\left(\mathfrak{g}_{0}\right)$.

Having given a fixed choice of a bundle $\mathscr{L}^{\lambda}$ of scales, a (local) scale on $M$ is a (local) smooth section of $\mathscr{L}^{\lambda}$.

Proposition 3.2. Let $G$ be a fixed semisimple Lie group, whose Lie algebra $\mathrm{g}$ is endowed with a $|k|$-grading. Then the following holds:

(1) There are scaling elements in $\precsim\left(\mathfrak{g}_{0}\right)$.

(2) Any scaling element $E_{\lambda} \in z\left(\mathfrak{g}_{0}\right)$ gives rise to a canonical bundle $\mathscr{L}^{\lambda}$ of scales over each manifold endowed with a parabolic geometry of the given type.

(3) Any bundle of scales admits global smooth sections, i.e. there always exist global scales.

Proof. (1) The grading element $E \in z\left(\mathfrak{g}_{0}\right)$, cf. 2.2 , acts on $\mathfrak{g}_{i}$ by multiplication with $i$, so it is a scaling element. More generally, one can consider the subspace of $z\left(g_{0}\right)$ of all elements which act by real scalars on each irreducible component of $\mathfrak{p}_{+}$. Then each irreducible component determines a real valued functional and thus a hyperplane in that space, and the complement of these finitely many hyperplanes (which is open and dense) consists entirely of scaling elements.

(2) Let $\mathfrak{p}_{+}=\oplus \mathfrak{p}^{\alpha}$ be the decomposition of $\mathfrak{p}_{+}$into $G_{0}$-irreducible components, and for a fixed grading element $E_{\lambda}$ denote by $a_{\alpha}$ the scalar by which $E_{\lambda}$ acts on $\mathfrak{p}^{\alpha}$. The adjoint action defines a smooth homomorphism $G_{0} \rightarrow \prod_{\alpha} G L\left(\mathfrak{p}^{\alpha}\right)$, whose components we write as $g \mapsto \operatorname{Ad}^{\alpha}(g)$. Then consider the homomorphism $\lambda: G_{0} \rightarrow \mathrm{R}^{+}$defined by

$$
\lambda(g):=\prod_{\alpha}\left|\operatorname{det}\left(\operatorname{Ad}^{\alpha}(g)\right)\right|^{2 a_{\alpha}} .
$$

The derivative of this homomorphism is given by $\lambda^{\prime}(A)=\sum_{\alpha} 2 a_{\alpha} \operatorname{tr}\left(\left.\operatorname{ad}(A)\right|_{\mathfrak{p}^{\alpha}}\right)$. Now $\mathfrak{g}_{-}=\oplus_{\alpha}\left(\mathfrak{p}^{\alpha}\right)^{*}$, and $E_{\lambda}$ acts on $\left(\mathfrak{p}^{\alpha}\right)^{*}$ by $-a_{\alpha}$ and on $\mathfrak{g}_{0}$ by zero, and thus $B\left(E_{\lambda}, A\right)=\operatorname{tr}\left(\operatorname{ad}(A) \circ \operatorname{ad}\left(E_{\lambda}\right)\right)=\sum_{\alpha} a_{\alpha} \operatorname{tr}\left(\left.\operatorname{ad}(A)\right|_{\mathfrak{p}^{\alpha}}\right)-\sum_{\alpha} a_{\alpha} \operatorname{tr}\left(\left.\operatorname{ad}(A)\right|_{\left(\mathfrak{p}^{\alpha}\right)^{*}}\right)$ $=\lambda^{\prime}(A)$. 
(3) This is just due to the fact that orientable real line bundles and thus principal $\mathbf{R}^{+}$-bundles are automatically trivial and hence admit global smooth sections.

Lemma 3.3. Let $\sigma: \mathscr{G}_{0} \rightarrow \mathscr{G}$ be a Weyl-structure for parabolic geometry $(\mathscr{G} \rightarrow M, \omega)$ and let $\mathscr{L}^{\lambda}$ be a bundle of scales.

(1) The Weyl connection $\sigma^{*} \omega_{0} \in \Omega_{1}\left(\mathscr{G}_{0}, \mathfrak{g}_{0}\right)$ induces a principal connection on the bundle of scales $\mathscr{L}^{\lambda}$.

(2) $\mathscr{L}^{\lambda}$ is naturally identified with $\mathscr{G}_{0} / \operatorname{ker}(\lambda)$, the orbit space of the free right action of the normal subgroup $\operatorname{ker}(\lambda) \subset G_{0}$ on $\mathscr{G}_{0}$.

(3) The form $\lambda^{\prime} \circ \sigma^{*} \omega_{0} \in \Omega^{1}\left(\mathscr{G}_{0}\right)$ descends to the connection form of the induced principal connection on $\mathscr{L}^{\lambda}=\mathscr{G}_{0} / \operatorname{ker}(\lambda)$.

(4) The composition of $\lambda^{\prime}$ with the curvature form of $\sigma^{*} \omega_{0}$ descends to the curvature of the induced connection on $\mathscr{L}^{\lambda}$.

PROOF. All claims are straightforward consequences of the definitions.

To see that the Weyl-structure $\sigma$ is actually uniquely determined by the induced principal connection on $\mathscr{L}^{\lambda}$ (cf. Theorem 3.8 below), we have to compute how the principal connection $\sigma^{*} \omega_{0}$ changes when we change $\sigma$. For later use, we also compute how the other objects induced by $\sigma$ change under the change of the Weyl-structures. So let us assume that $\hat{\sigma}$ is another Weylstructure and $\Upsilon=\left(\Upsilon_{1}, \ldots, \Upsilon_{k}\right)$ is the section of $\mathscr{A}_{1} \oplus \cdots \oplus \mathscr{A}_{k}$ characterized by $\hat{\sigma}(u)=\sigma(u) \exp \left(\Upsilon_{1}(u)\right) \ldots \exp \left(\Upsilon_{k}(u)\right)$.

We shall use the convention that we simply denote quantities corresponding to $\hat{\sigma}$ by hatted symbols and quantities corresponding to $\sigma$ by unhatted symbols. Consequently, $\left(\xi_{-k}, \ldots, \xi_{-1}\right)$ and $\left(\hat{\xi}_{-k}, \ldots, \hat{\xi}_{-1}\right)$ denote the splitting of $\xi \in$ $T M$ according to $\sigma$, respectively $\hat{\sigma}$, and $\mathbf{P}$ and $\hat{\mathbf{P}}$ are the Rho-tensors. Finally, let us consider any vector bundle $E$ associated to the principal bundle $\mathscr{G}_{0}$. Then for any Weyl-structure the corresponding principal connection on $\mathscr{G}_{0}$ induces a linear connection on $E$, which is denoted by $\nabla$ for $\sigma$ and by $\hat{\nabla}$ for $\hat{\sigma}$.

To write the formulae efficiently, we need some further notation. By $j$ we denote a sequence $\left(j_{1}, \ldots, j_{k}\right)$ of nonnegative integers, and we put $\|\underline{j}\|=j_{1}+$ $2 j_{2}+\cdots+k j_{k}$. Moreover, we define $j !=j_{1} ! \ldots j_{k}$ ! and $(-1)^{\underline{j}}=(-\overline{1})^{j_{1}+\cdots+j_{k}}$, and we define $(\underline{j})_{m}$ to be the subsequence $\left(j_{1}, \ldots, j_{m}\right)$ of $\underline{j}$. By 0 we denote sequences of any length consisting entirely of zeros.

Proposition 3.4. Let $\sigma$ and $\hat{\sigma}$ be two Weyl-structures related by

$$
\hat{\sigma}(u)=\sigma(u) \exp \left(\Upsilon_{1}(u)\right) \ldots \exp \left(\Upsilon_{k}(u)\right)
$$


where $\Upsilon=\left(\Upsilon_{1}, \ldots, \Upsilon_{k}\right)$ is a smooth section of $\mathscr{A}_{1} \oplus \cdots \oplus \mathscr{A}_{k}$. Then we have:

$$
\hat{\xi}_{i}=\sum_{\|\underline{j}\|+\ell=i} \frac{(-1)^{\underline{j}}}{\underline{j} !} \operatorname{ad}\left(\Upsilon_{k}\right)^{j_{k}} \circ \cdots \circ \operatorname{ad}\left(\Upsilon_{1}\right)^{j_{1}}\left(\xi_{\ell}\right),
$$

(2) $\hat{\mathrm{P}}_{i}(\xi)=\sum_{\|\underline{j}\|+\ell=i} \frac{(-1)^{\underline{j}}}{\underline{j} !} \operatorname{ad}\left(\Upsilon_{k}\right)^{j_{k}} \circ \cdots \circ \operatorname{ad}\left(\Upsilon_{1}\right)^{j_{1}}\left(\xi_{\ell}\right)$

$$
\begin{aligned}
& +\sum_{\|\underline{j}\|+\ell=i} \frac{(-1)^{\underline{j}}}{\underline{j} !} \operatorname{ad}\left(\Upsilon_{k}\right)^{j_{k}} \circ \cdots \circ \operatorname{ad}\left(\Upsilon_{1}\right)^{j_{1}}\left(\mathrm{P}_{\ell}(\xi)\right) \\
& +\sum_{m=1}^{k} \sum_{\substack{j j_{m-1}=0 \\
m+\|\underline{j}\|=i}} \frac{(-1)^{\underline{j}}}{\left(j_{m}+1\right) \underline{j} !} \operatorname{ad}\left(\Upsilon_{k}\right)^{j_{k}} \circ \cdots \circ \operatorname{ad}\left(\Upsilon_{m}\right)^{j_{m}}\left(\nabla_{\xi} \Upsilon_{m}\right),
\end{aligned}
$$

where ad denotes the adjoint action with respect to the algebraic bracket $\{$,$\} .$

If $E$ is an associated vector bundle to the principal bundle $\mathscr{G}_{0}$, then we have:

$$
\hat{\nabla}_{\xi} s=\nabla_{\xi} s+\sum_{\|\underline{j}\|+\ell=0} \frac{(-1)^{\underline{j}}}{\underline{j} !}\left(\operatorname{ad}\left(\Upsilon_{k}\right)^{j_{k}} \circ \cdots \circ \operatorname{ad}\left(\Upsilon_{1}\right)^{j_{1}}\left(\xi_{\ell}\right)\right) \bullet s,
$$

where $\bullet$ denotes the map $\mathscr{A}_{0} \times E \rightarrow E$ induced by the action of $\mathfrak{g}_{0}$ on the standard fiber of $E$.

Proof. The essential part of the proof is to compute the tangent map $T_{u} \hat{\sigma}$ in a point $u \in \mathscr{G}_{0}$. By definition, $\hat{\sigma}(u)=\sigma(u) \exp \left(\Upsilon_{1}(u)\right) \ldots \exp \left(\Upsilon_{k}(u)\right)$. Thus, we can write the evaluation of the tangent map, $T_{u} \hat{\sigma} \cdot \xi$, as the sum of $T_{\sigma(u)} r^{g} \cdot T_{u} \sigma \cdot \xi$, where $g=\exp \left(\Upsilon_{1}(u)\right) \ldots \exp \left(\Upsilon_{k}(u)\right) \in P_{+}$, and the derivative at $t=0$ of

$$
\sigma(u) \exp \left(\Upsilon_{1}(c(t))\right) \ldots \exp \left(\Upsilon_{k}(c(t))\right),
$$

where $c: \mathrm{R} \rightarrow \mathscr{G}_{0}$ is a smooth curve with $c(0)=u$ and $c^{\prime}(0)=\xi$. By construction, the latter derivative lies in the kernel of $T \pi$, where $\pi: \mathscr{G} \rightarrow \mathscr{G}_{0}$ is the projection, so we can write it as $\zeta_{\Phi(\xi)}(\hat{\sigma}(u))$ for suitable $\Phi(\xi) \in \mathfrak{p}_{+}$.

Now, for $\xi \in T_{u} \mathscr{G}_{0}$, we have $\hat{\sigma}^{*} \omega(\xi)=\omega(\hat{\sigma}(u))\left(T_{u} \hat{\sigma} \cdot \xi\right)$. By equivariance of the Cartan connection $\omega$, we get $\omega(\sigma(u) \cdot g)\left(\operatorname{Tr}^{g} \cdot T \sigma \cdot \xi\right)=\operatorname{Ad}\left(g^{-1}\right)(\omega(u)$ $(T \sigma \cdot \xi))$. Consequently,

$$
\hat{\sigma}^{*} \omega(\xi)=\operatorname{Ad}\left(g^{-1}\right)\left(\sigma^{*} \omega(\xi)\right)+\Phi(\xi) .
$$

Since $\Phi(\xi) \in \mathfrak{p}_{+}$, this term affects only the transformation of the Rho-tensor, and does not influence the changes of $\sigma^{*} \omega_{i}$ for $i \leq 0$. In particular, for the 
components $\hat{\sigma}^{*} \omega_{i}$ with $i<0$, we only have to take the part of the right degree in

$$
e^{\operatorname{ad}\left(-\Upsilon_{k}(u)\right)} \circ \cdots \circ e^{\operatorname{ad}\left(-\Upsilon_{1}(u)\right)}\left(\sigma^{*} \omega(u)(\xi)\right),
$$

and expanding the exponentials, this immediately leads to formula (1).

To compute the change in the connection, we have to notice that $\hat{\sigma}^{*} \omega_{0}(\xi)$ is the component of degree zero in (4) above. Consequently, if we apply $\hat{\sigma}^{*} \omega_{0}$ to the horizontal lift of a tangent vector on $M$, the outcome is just this degree zero part. Otherwise put, the horizontal lift with respect to $\hat{\sigma}^{*} \omega_{0}$ is obtained by subtracting the fundamental vector field corresponding to the degree zero part of (4) from the horizontal lift with respect to $\sigma^{*} \omega_{0}$. Applying such horizontal vector field to a smooth $G_{0}$-equivariant function with values in any $G_{0}$-representation and taking into account that a fundamental vector fields acts on such functions by the negative of its generator acting on the values, this immediately leads to formula (3) by expanding the exponentials.

Finally, we have to deal with the change of the Rho-tensor. Recall that we view this as a tensor on the manifold $M$, so we can compute $\hat{\mathrm{P}}_{i}(\xi)$ by applying $\hat{\sigma}^{*} \omega_{i}$ to any lift of $\xi$. In particular, we may use the horizontal lift $\xi^{h}$ with respect to $\sigma^{*} \omega_{0}$, so we may assume $\sigma^{*} \omega_{0}(\xi)=0$. But then expanding the exponentials in (4) and taking the part of degree $i$ we see that we exactly get the first two summands in formula (2). Thus we are left with proving that the last summand corresponds to $\Phi(\xi)$. For this aim, let us rewrite the curve that we have to differentiate as

$$
\hat{\sigma}(u) \exp \left(-\Upsilon_{k}(u)\right) \ldots \exp \left(-\Upsilon_{1}(u)\right) \exp \left(\Upsilon_{1}(c(t))\right) \ldots \exp \left(\Upsilon_{k}(c(t))\right) .
$$

Differentiating this using the product rule we get a sum of terms in which one $\Upsilon_{i}$ is differentiated, while all others have to be evaluated at $t=0$, i.e. in $u$. So each of these terms reads as the derivative at $t=0$ of

$$
\hat{\sigma}(u) \cdot \operatorname{conj}_{\exp \left(-\Upsilon_{k}(u)\right)} \circ \cdots \circ \operatorname{conj}_{\exp \left(-\Upsilon_{i+1}(u)\right)}\left(\exp \left(-\Upsilon_{i}(u)\right) \exp \left(\Upsilon_{i}(c(t))\right)\right),
$$

where $\operatorname{conj}_{g}$ denotes the conjugation by $g$, i.e. the map $h \mapsto g h g^{-1}$. This expression is just the principal right action by the value of a smooth curve in $P$ which maps zero to the unit element, so its result is exactly the value at $\hat{\sigma}(u)$ of the fundamental vector field generated by the derivative at zero of this curve. This derivative is clearly obtained by applying

$$
e^{\operatorname{ad}\left(-\Upsilon_{k}(u)\right)} \circ \cdots \circ e^{\operatorname{ad}\left(-\Upsilon_{i+1}(u)\right)}
$$

to the derivative at zero of $t \mapsto \exp \left(-\Upsilon_{i}(u)\right) \exp \left(\Upsilon_{i}(c(t))\right)$. By [20], 4.26, and the chain rule, the latter derivative equals the left logarithmic derivative 
of exp applied to the derivative at zero of $t \mapsto \Upsilon_{i}(c(t))$. Moreover, the proof of [20], Lemma 4.27, can be easily adapted to the left logarithmic derivative, showing that this gives

$$
\sum_{p=0}^{\infty} \frac{(-1)^{p}}{(p+1) !} \operatorname{ad}\left(\Upsilon_{i}(u)\right)^{p}\left(\xi^{h} \cdot \Upsilon_{i}\right)
$$

Finally, we have to observe that $\xi^{h} \cdot \Upsilon_{i}$ corresponds to $\nabla_{\xi} \Upsilon_{i}$ and to sort out the terms of the right degree in order to get the remaining summand in (2).

\subsection{Example}

For all irreducible parabolic geometries, the formulae from Proposition 3.4 become extremely simple. In fact they coincide completely with the known ones in the conformal Riemannian geometry: The grading of $T M$ is trivial, the connection transforms as

$$
\hat{\nabla}_{\xi} s=\nabla_{\xi} s-\{\Upsilon, \xi\} \bullet s,
$$

where $\Upsilon$ is a section of $\mathscr{A}_{1}=T^{*} M$, and the bracket of $\Upsilon$ and $\xi$ is a field of endomorphisms of $T M$ acting on $s$ in an obvious way. Indeed, there are no more terms on the right-hand side of 3.4(3) which make sense. Next, the Rho-tensor transforms as

$$
\hat{\mathrm{P}}(\xi)=\mathbf{P}(\xi)+\nabla_{\xi} \Upsilon+\frac{1}{2}\{\Upsilon,\{\Upsilon, \xi\}\} .
$$

The formulae for the $|2|$-graded examples are a bit more complicated. The splitting of $T M$ and the connection and Rho-tensors change as follows

$$
\begin{aligned}
\hat{\xi}_{-2}= & \xi_{-2} \\
\hat{\xi}_{-1}= & \xi_{-1}-\left\{\Upsilon_{1}, \xi_{-2}\right\} \\
\hat{\nabla}_{\xi} s= & \nabla_{\xi} s+\left(\frac{1}{2}\left\{\Upsilon_{1},\left\{\Upsilon_{1}, \xi \xi_{-2}\right\}\right\}-\left\{\Upsilon_{2}, \xi \xi_{-2}\right\}-\left\{\Upsilon_{1}, \xi_{-1}\right\}\right) \bullet s, \\
\hat{\mathrm{P}}_{1}(\xi)= & \mathrm{P}_{1}(\xi)-\frac{1}{6}\left\{\Upsilon_{1},\left\{\Upsilon_{1},\left\{\Upsilon_{1}, \xi_{-2}\right\}\right\}\right\}+\left\{\Upsilon_{2},\left\{\Upsilon_{1}, \xi_{-2}\right\}\right\} \\
& \quad+\frac{1}{2}\left\{\Upsilon_{1},\left\{\Upsilon_{1}, \xi_{-1}\right\}\right\}-\left\{\Upsilon_{2}, \xi_{-1}\right\}+\nabla_{\xi} \Upsilon_{1} \\
\hat{\mathrm{P}}_{2}(\xi)= & \mathrm{P}_{2}(\xi)-\left\{\Upsilon_{1}, \mathrm{P}_{1}(\xi)\right\}+\nabla_{\xi} \Upsilon_{2}-\frac{1}{2}\left\{\Upsilon_{1}, \nabla_{\xi} \Upsilon_{1}\right\} \\
& \quad+\frac{1}{24} \operatorname{ad}\left(\Upsilon_{1}\right)^{4}\left(\xi_{-2}\right)-\frac{1}{2}\left\{\Upsilon_{2},\left\{\Upsilon_{1},\left\{\Upsilon_{1}, \xi_{-2}\right\}\right\}\right\}+\frac{1}{2}\left\{\Upsilon_{2},\left\{\Upsilon_{2}, \xi_{-2}\right\}\right\} \\
& \quad-\frac{1}{6} \operatorname{ad}\left(\Upsilon_{1}\right)^{3}\left(\xi_{-1}\right)+\left\{\Upsilon_{2},\left\{\Upsilon_{1}, \xi_{-1}\right\}\right\} .
\end{aligned}
$$




\subsection{Remark}

In applications, one is often interested in questions about the dependence of some objects on the choice of the Weyl-structures and then the infinitesimal form of the available change of the splittings, Rho's and connections is important. In our terms, this amounts to sorting out the terms in formulae 3.4(1)-(3) which are linear in upsilons. Thus, the infinitesimal version of Proposition 3.4 for the variations $\delta \xi_{i}, \delta \nabla$, and $\delta \mathbf{P}_{i}$ reads

$$
\begin{aligned}
\delta \xi_{i}= & -\left\{\Upsilon_{1}, \xi_{i-1}\right\}-\cdots-\left\{\Upsilon_{k+i}, \xi_{-k}\right\} \\
\delta \mathrm{P}_{i}(\xi)= & \nabla_{\xi} \Upsilon_{i}-\left\{\Upsilon_{1}, \mathrm{P}_{i-1}(\xi)\right\}-\cdots-\left\{\Upsilon_{i-1}, \mathrm{P}_{1}(\xi)\right\} \\
& -\left\{\Upsilon_{i+1}, \xi_{-1}\right\}-\cdots-\left\{\Upsilon_{k}, \xi_{-k+i}\right\} \\
\delta \nabla_{\xi} s= & -\left(\left\{\Upsilon_{1}, \xi_{-1}\right\}+\ldots+\left\{\Upsilon_{k}, \xi_{-k}\right\}\right) \bullet s .
\end{aligned}
$$

3.8.

Proposition 3.4 not only allows us to show that a Weyl-structure is uniquely determined by the induced connection on any bundle of scales, but it also leads to a description of the Cartan bundle $p: \mathscr{G} \rightarrow M$. To get this description, recall that for any principal bundle $E \rightarrow M$ there is a bundle $Q E \rightarrow M$ whose sections are exactly the principal connections on $E$, see [20], 17.4.

THeOREM. Let $p: \mathscr{G} \rightarrow M$ be a parabolic geometry on $M$, and let $\mathscr{L}^{\lambda} \rightarrow M$ be a bundle of scales.

(1) Each Weyl-structure $\sigma: \mathscr{G}_{0} \rightarrow \mathscr{G}$ determines the principal connection on $\mathscr{L}^{\lambda}$ induced by the Weyl connection $\sigma^{*} \omega_{0}$. This defines a bijective correspondence between the set of Weyl-structures and the set of principal connections on $\mathscr{L}^{\lambda}$.

(2) There is a canonical isomorphism $\mathscr{G} \cong p_{0}^{*} Q \mathscr{L}^{\lambda}$, where $p_{0}: \mathscr{G}_{0} \rightarrow M$ is the projection. Under this isomorphism, the choice of a Weyl structure $\sigma: \mathscr{G}_{0} \rightarrow \mathscr{G}$ is the pullback of the principal connection on the bundle of scales $\mathscr{L}^{\lambda}$, viewed as a section $M \rightarrow Q \mathscr{L}^{\lambda}$. Moreover, the principal action of $G_{0}$ is the canonical action on $p_{0}^{*} Q \mathscr{L}^{\lambda}$ induced from the action on $\mathscr{G}_{0}$, while the action of $P_{+}$is described by equation (3) from Proposition 3.4.

Proof. (1) Consider the map $\lambda^{\prime}: \mathfrak{g}_{0} \rightarrow \mathrm{R}$ defining the bundle $\mathscr{L}^{\lambda}$ of scales. Take elements $Z \in \mathfrak{p}_{+}$and $X \in \mathfrak{g}_{-}$, and consider $\lambda^{\prime}([Z, X])$. By assumption, this is given by $B\left(E_{\lambda},[Z, X]\right)=B\left(\left[E_{\lambda}, Z\right], X\right)$ for some scaling element $E_{\lambda} \in \jmath\left(\mathfrak{g}_{0}\right)$. Hence if we assume that $Z$ lies in a $G_{0}$-irreducible component of $\mathfrak{p}_{+}$this is just a nonzero real multiple of $B(Z, X)$. In particular, this implies that for each $0 \neq Z \in \mathfrak{p}_{i}$, we can find an element $X \in \mathfrak{g}_{-i}$, such that 


\section{Cartan connection $\omega$}

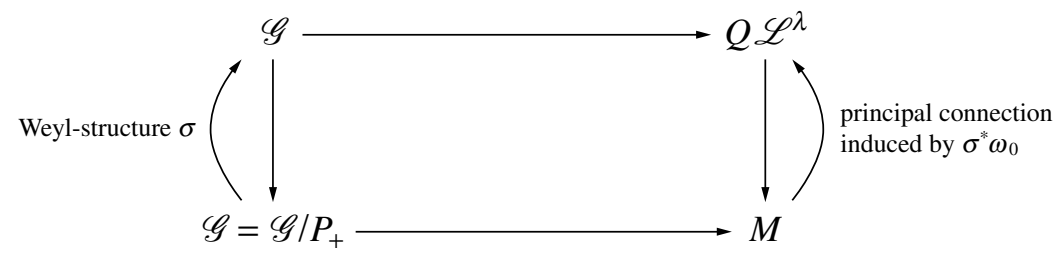

soldering form $\sigma^{*} \omega_{-} \in \Omega^{1}\left(\mathscr{G}_{0}, \mathfrak{g}_{-}\right)$

Weyl connection $\sigma^{*} \omega_{0} \in \Omega^{1}\left(\mathscr{G}_{0}, \mathfrak{g}_{0}\right)$

Rho-tensor $\mathrm{P}=\sigma^{*} \omega_{+} \in \Omega^{1}\left(\mathscr{G}_{0}, \mathfrak{g}_{+}\right)$

$\sigma^{*} \omega_{-} \in \Omega^{1}\left(M ; \mathrm{Gr} \mathscr{A}_{-}\right)$

$\mathrm{P} \in \Omega^{1}\left(M ; \mathrm{Gr} \mathscr{A}_{+}\right)$

FIGURE 1. Pullback diagram with further objects related to Weyl-structures

$\lambda^{\prime}([Z, X]) \neq 0$. Moreover, since $E_{\lambda} \in z\left(g_{0}\right)$ we get $\operatorname{Ad}(g)\left(E_{\lambda}\right)=E_{\lambda}$ for all $g \in G_{0}$ and this immediately implies that mapping $Z \in \mathfrak{g}_{i}$ to $X \mapsto \lambda^{\prime}([Z, X])$ induces an isomorphism $\mathfrak{g}_{i} \cong \mathrm{g}_{-i}^{*}$ of $G_{0}$-modules.

To prove (1), we may as well use the induced linear connection on the line bundle $L^{\lambda}=\mathscr{L}^{\lambda} \times_{\mathrm{R}^{+}} \mathrm{R}$ corresponding to the standard representation. For this bundle, the map $\bullet$ from Proposition 3.4 is clearly given by $(A \bullet s)(x)=$ $\lambda^{\prime}(A(x)) s(x)$, where we denote by $\lambda^{\prime}: \mathscr{A}_{0} \rightarrow M \times \mathrm{R}$ also the mapping induced by $\lambda^{\prime}: \mathrm{g}_{0} \rightarrow \mathrm{R}$.

We first claim that the map from Weyl-structures to linear connections is injective. So assume that $\sigma$ and $\hat{\sigma}$ induce the same linear connection on $L^{\lambda}$ and let $\Upsilon$ be the section of $\mathscr{A}_{1} \oplus \cdots \oplus \mathscr{A}_{k}$ describing the change from $\sigma$ to $\hat{\sigma}$. For $\xi \in T^{-1} M$, we have $\xi_{i}=0$ for all $i<-1$, hence formula (3) of Proposition 3.4 reduces to $\hat{\nabla}_{\xi} s=\nabla_{\xi} s+\lambda^{\prime}\left(\left\{\Upsilon_{1}, \xi\right\}\right) s$ in this case. If $\Upsilon_{1}$ would be nonzero, then by the above argument we could find $\xi$ such that $\lambda^{\prime}\left(\left\{\Upsilon_{1}, \xi\right\}\right) \neq 0$, which would contradict $\hat{\nabla}=\nabla$, so $\Upsilon_{1}$ must be identically zero. But then for $\xi$ in $T^{-2} M$, the change reduces to $\hat{\nabla}_{\xi} s=\nabla_{\xi} s+\lambda^{\prime}\left(\left\{\Upsilon_{2}, \xi_{-2}\right\}\right) s$ and as above, we conclude that $\Upsilon_{2}$ is identically zero. Inductively, we get $\Upsilon=0$ and thus $\hat{\sigma}=\sigma$.

To see surjectivity, assume that $\hat{\nabla}$ is any linear connection on $L^{\lambda}$, and let $\sigma$ be any Weyl-structure with induced linear connection $\nabla$ on $L^{\lambda}$. Then there is a one-form $\tau \in \Omega^{1}(M)$ such that $\hat{\nabla}_{\xi}=\nabla_{\xi} s+\tau(\xi) s$. Restricting $\tau$ to $T^{-1} M$, we can find a unique smooth section $\Upsilon_{1}$ of $\mathscr{A}_{1}$ such that $\tau(\xi)=-\lambda^{\prime}\left(\left\{\Upsilon_{1}, \xi\right\}\right)$ for all $\xi$ in $T^{-1} M$. Next, consider the map $T^{-2} M \rightarrow M \times \mathrm{R}$ given by

$$
\xi \mapsto \tau(\xi)+\lambda^{\prime}\left(\left\{\Upsilon_{1}, \xi_{-1}\right\}\right)-\frac{1}{2} \lambda^{\prime}\left(\left\{\Upsilon_{1},\left\{\Upsilon_{1}, \xi_{-2}\right\}\right\}\right),
$$


where the $\xi_{i}$ are the components of $\xi$ with respect to the Weyl-structure $\sigma$. By construction, this vanishes on $T^{-1} M$, so it factors to a map defined on $\mathscr{A}_{-2}$, and thus there is a unique section $\Upsilon_{2}$ of $\mathscr{A}_{2}$ such that it equals $-\lambda^{\prime}\left(\left\{\Upsilon_{2}, \xi_{-2}\right\}\right)$. Inductively, we find a section $\Upsilon$ such that the Weyl-structure $\hat{\sigma}$ corresponding to $\sigma$ and $\Upsilon$ induces the linear connection $\hat{\nabla}$, cf. formula 3.4(3).

(2) Consider any point $u \in \mathscr{G}$. Proposition 3.1 implies that there is a Weylstructure $\sigma: \mathscr{G}_{0} \rightarrow \mathscr{G}$ such that $u=\sigma(\pi(u))$. If $\nabla$ is the linear connection on $L^{\lambda}$ induced by $\sigma$, then we see from Proposition 3.4 that the value of $\nabla_{\xi} s(p(u))$ for a vector field $\xi$ on $M$ and a section $s$ of $L^{\lambda}$ depends only on $\sigma(p(u))$, since its change under a change of the Weyl-structure depends only on the value of $\Upsilon$ in $p(u)$. Thus, mapping $u$ to the value at $p(u)$ of the principal connection on $\mathscr{L}^{\lambda}$ induced by $\sigma^{*} \omega_{0}$ is independent of the choice of $\sigma$, so we get a well defined bundle map from the bundle $\mathscr{G} \rightarrow \mathscr{G}_{0}$ to the bundle $Q \mathscr{L}^{\lambda} \rightarrow M$ covering the projection $p_{0}: \mathscr{G}_{0} \rightarrow M$. Moreover, from part (1) of this proof it follows that this map induces isomorphisms in each fiber, so it leads to an isomorphism $\mathscr{G} \rightarrow p_{0}^{*} Q \mathscr{L}^{\lambda}$ of bundles over $\mathscr{G}_{0}$. Obviously, the $G_{0}$-equivariant sections of $p_{0}^{*} Q \mathscr{L}^{\lambda} \rightarrow \mathscr{G}_{0}$ correspond exactly to the induced principal connections on $\mathscr{L}^{\lambda}$, i.e. the sections of $Q \mathscr{L}^{\lambda} \rightarrow M$.

In order to describe the principal action of $P$ on $p_{0}^{*} Q \mathscr{L}^{\lambda}$ obtained by the above isomorphism, one just has to note that for $u \in \mathscr{G}_{0}$ and $g \in G_{0}$ the fibers of $p_{0}^{*} Q \mathscr{L}^{\lambda}$ over $u$ and $u \cdot g$ are canonically isomorphic since $p_{0}(u)=p_{0}(u \cdot g)$. Thus, the principal right action of $G_{0}$ is simply given by acting on $\mathscr{G}_{0}$. On the other hand, fix $u \in \mathscr{G}_{0}$ and an element $\exp \left(Z_{1}\right) \ldots \exp \left(Z_{k}\right) \in P_{+}$for $Z_{i} \in \mathfrak{g}_{i}$. Via $u$, the element $Z_{i}$ corresponds to an element $\Upsilon_{i} \in \mathscr{A}_{i}$ at the point $p_{0}(u)$. Then the principal right action of $P_{+}$is described by the formula (3) of Proposition 3.4 as required.

\subsection{Closed and exact Weyl-structures}

Let us fix a bundle of scales $\mathscr{L}^{\lambda}$ for some parabolic geometry. Then the bijective correspondence between Weyl-structures and principal connections on $\mathscr{L}^{\lambda}$ immediately leads to two distinguished subclasses of Weyl-structures. Namely, we call a Weyl-structure $\sigma: \mathscr{G}_{0} \rightarrow \mathscr{G}$ closed, if the induced principal connection on $\mathscr{L}^{\lambda}$ (or equivalently the induced linear connection $\nabla$ on $L^{\lambda}$ ) is flat.

Moreover, by Proposition 3.2 the bundle $\mathscr{L}^{\lambda}$ of scales admits global smooth sections, and any such section gives rise to a flat principal connection on $\mathscr{L}^{\lambda}$ (which in addition has trivial holonomy) and hence to a closed Weyl-structure. The closed Weyl-structures induced by such global sections are called exact.

Note that in the case of conformal structures, the canonical choice for the bundle of scales is simply the $\mathrm{R}^{+}$-bundle whose smooth sections are the metrics 
in the conformal class. Thus, the exact Weyl-structures in conformal geometry correspond exactly to the Levi-Civita connections of the metrics in the conformal class.

The reason for the names "closed" and "exact" becomes apparent, once one studies the affine structures on the sets of closed and exact Weyl-structures. So let us assume that $\sigma$ is a closed Weyl-structure, and consider any other Weylstructure $\hat{\sigma}$ corresponding to the section $\Upsilon=\left(\Upsilon_{1}, \ldots, \Upsilon_{k}\right)$ of $\mathscr{A}_{1} \oplus \cdots \oplus$ $\mathscr{A}_{k}$. Now we can reinterpret theorem 3.8(1) together with proposition 3.4 as showing that the set of Weyl-structures is an affine space over $\Omega^{1}(M)$, in such a way that fixing $\sigma$ the section $\Upsilon$ corresponds to the one-form $\Upsilon^{\sigma, \lambda}$ defined by

$$
\Upsilon^{\sigma, \lambda}(\xi)=\sum_{\|\underline{j}\|+\ell=0} \frac{(-1)^{j}}{\underline{j} !} \lambda^{\prime}\left(\operatorname{ad}\left(\Upsilon_{k}\right)^{j_{k}} \circ \cdots \circ \operatorname{ad}\left(\Upsilon_{1}\right)^{j_{1}}\left(\xi_{\ell}\right)\right) .
$$

This identification is obtained simply by pulling back the affine structure on the space of principal connections on $\mathscr{L}^{\lambda}$ to the space of Weyl-structures. In particular, the change of the principal connections $\tau$ and $\hat{\tau}$ on $\mathscr{L}^{\lambda}$ induced by $\sigma$ and $\hat{\sigma}$, respectively, is just given by $\hat{\tau}=\tau+\Upsilon^{\sigma, \lambda}$. But then their curvatures change simply by $\hat{\rho}=\rho+d \Upsilon^{\sigma, \lambda}$, so in particular if $\sigma$ is closed then $\hat{\sigma}$ is closed if and only if $d \Upsilon^{\sigma, \lambda}=0$. Thus, in the same way as Weyl-structures are affine over all one-forms, closed Weyl-structures are affine over closed one-forms.

For exact Weyl-structures, the situation is even simpler. If $s$ and $\hat{s}$ are two global sections of $\mathscr{L}^{\lambda}$, then there is a unique smooth function $f$ such that $\hat{s}(x)=e^{-f(x)} s(x)$. It is then well known that the associated principal connections simply change by $\hat{\tau}=\tau+d f$, so exact Weyl-structures are affine over the space of exact one-forms.

\subsection{Remark}

Another useful observation about exact Weyl geometries is related to the identification of $\mathscr{L}^{\lambda}$ with $\mathscr{G}_{0} / \operatorname{ker} \lambda$ from Lemma 3.3(2). By the general properties of classical $G$-structures, the sections of such bundles are in bijective correspondence with reductions of the structure groups to ker $\lambda \subset G_{0}$. Thus the holonomy of the Weyl connections given by exact Weyl structures is always at most ker $\lambda$. In particular, in $|1|$-graded cases the scaling element is unique up to scalar multiples, and the kernel of $\lambda$ is exactly the semisimple part of $G_{0}$. The same observation is then true for the closed Weyl geometries locally.

\subsection{Normal Weyl-structures}

Besides the rather obvious closed and exact Weyl-structures discussed above there is a second kind of special Weyl-structures, the so-called normal Weylstructures. In several respects, they are quite different from closed and exact 
Weyl-structures. On one hand, they are "more canonical" since their definition does not involve the choice of a bundle of scales. On the other hand, in contrast to closed and exact Weyl-structures, which always exist globally, normal Weylstructures in general exist only locally (over $M$ ). Their existence is closely related to the existence of normal coordinates for parabolic geometries. This subject will be taken up elsewhere. We would like to point out at this place that the existence of normal Weyl-structures seems to be a new result even in the case of conformal structures, where it significantly improves the result on the existence of Graham normal coordinates, see [24].

Since the Rho tensors give the information about the difference of the covariant derivative with respect to the Weyl connection and the invariant derivative with respect to $\omega$ along the image of the chosen Weyl-structure $\sigma$, the "normality" we have in mind will be described in terms of certain minimality of $P$. More explicitly, a Weyl-structure $\sigma$ will be called normal at the point $x \in M$ if it satisfies the properties imposed in Theorem 3.5. This Theorem also describes completely the freedom in the choice.

Recall that once we have chosen a Weyl-structure, we get an identification of the tangent bundle with its associated graded vector bundle. Thus $T M$ is associated to $\mathscr{G}_{0}$ and so there is the induced linear Weyl connection on $T M$. Since the Weyl-structure induces covariant derivatives on all components of the associated graded of the tangent bundle, the Weyl connection on $T M$ preserves the grading. For the same reason, we can form covariant derivatives of the Rho-tensor, viewed as a one-form with values in $T^{*} M \cong \mathscr{A}_{1} \oplus \cdots \oplus \mathscr{A}_{k}$, which again preserve the grading.

THEOREM 3.5. Let $p: \mathscr{G} \rightarrow M$ be a parabolic geometry with underlying $G_{0}$-bundle $p_{0}: \mathscr{G}_{0} \rightarrow M$ and let $\pi: \mathscr{G} \rightarrow \mathscr{G}_{0}$ be the canonical projection. Let $x \in M$ be a point and let $u_{0} \in \mathscr{G}_{0}$ and $u \in \mathscr{G}$ be points such that $\pi(u)=u_{0}$ and $p_{0}\left(u_{0}\right)=x$. Then there exists an open neighborhood $U$ of $x$ in $M$ and a Weyl-structure $\sigma: p_{0}^{-1}(U) \rightarrow p^{-1}(U)$ such that $\sigma\left(u_{0}\right)=u$ and the Rhotensor $\mathrm{P}$ of $\sigma$ has the property that for all $k \in \mathrm{N}$ the symmetrization over all $\xi_{i}$ of $\left(\nabla_{\xi_{k}} \ldots \nabla_{\xi_{1}} \mathrm{P}\right)\left(\xi_{0}\right)$ vanishes at $x$, so in particular $\mathrm{P}(x)=0$. Moreover, this condition uniquely determines the infinite jet of $\sigma$ in $u_{0}$.

Proof. Consider the Cartan connection $\omega$ on $\mathscr{G}$. Since $\omega$ restricts to a linear isomorphism, for each element $A \in \mathfrak{g}$ we get the constant vector field $\tilde{A} \in \mathfrak{X}(\mathscr{G})$ defined by $\tilde{A}(v)=\omega(v)^{-1}(A)$, cf. 2.3. (Note that for $A \in \mathfrak{p}$ this is just the fundamental vector field.) In particular, we may consider the vector fields $\tilde{X}$ for $X \in \mathfrak{g}_{-}$. Now we can find a neighborhood $V$ of zero in $\mathfrak{g}_{-}$, such that for all $X \in V$ the flow of $X$ in the point $u$ exists up to time $t=1$. Define $\varphi: V \rightarrow \mathscr{G}$ by $\varphi(X)=\mathrm{Fl}_{1}^{\tilde{X}}(u)$. Since $T_{u} p \circ T_{0} \varphi: \mathfrak{g}_{-} \rightarrow T_{x} M$ is obviously a linear isomorphism, we may assume (possibly shrinking $V$ ) that 
the maps $\varphi, \pi \circ \varphi$ and $p \circ \varphi$ are all diffeomorphisms onto their images, and we put $U=p(\varphi(V))$. For a point $v_{0} \in p_{0}^{-1}(U)$ there clearly exist unique elements $X \in V$ and $g \in G_{0}$ such that $v_{0}=\pi(\varphi(X)) \cdot g$, and we define $\sigma\left(v_{0}\right):=\varphi(X) \cdot g$. Obviously, this defines a smooth $G_{0}$-equivariant section $\sigma: p_{0}^{-1}(U) \rightarrow p^{-1}(U)$ and $\sigma\left(u_{0}\right)=u$.

Next, consider a tangent vector $\xi \in T_{x} M$, and its horizontal lift $\xi^{h} \in T_{u_{0}} \mathscr{G}_{0}$ with respect to the principal connection $\sigma^{*} \omega_{0}$. Since $\sigma^{*} \omega_{\leq 0}$ defines a Cartan connection on $p_{0}^{-1}(U)$ (see 3.4) we can extend $\xi^{h}$ uniquely to a vector field $\tilde{\xi}^{h}$ such that $\sigma^{*} \omega_{\leq 0}\left(\tilde{\xi}^{h}\right)$ is constantly equal to some $X \in \mathfrak{g}_{-}$. Moreover, $\tilde{\xi}^{h}$ is projectable to a vector field $\tilde{\xi}$ on $U$ and it is exactly the horizontal lift of $\tilde{\xi}$ (which also justifies the notation).

Now consider the flow line $c(t)=\mathrm{Fl}_{t}^{\tilde{X}}(u)=\varphi(t X)$ in $\mathscr{G}$, which is defined for sufficiently small $t$. By construction, we have $\sigma(\pi(c(t)))=c(t)$ for all $t$. But this implies that $T \sigma \cdot(\pi \circ c)^{\prime}(t)=c^{\prime}(t)$, so $\sigma^{*}(\omega)\left((\pi \circ c)^{\prime}(t)\right)$ is constantly equal to $X$ and thus $(\pi \circ c)(t)=\mathrm{Fl}_{t}^{\tilde{\xi}}\left(u_{0}\right)$. On the other hand, from the construction it is clear that $\omega\left(c^{\prime}(t)\right)=X \in \mathfrak{g}_{-}$, so if we consider the function $\mathrm{P}: \mathscr{G}_{0} \rightarrow L\left(\mathfrak{g}_{-}, \mathfrak{p}_{+}\right)$describing the Rho-tensor, then $\mathrm{P}(\pi(c(t)))(X)=0$ for all $t$. Consequently, all derivatives of this curve in $t=0$ vanish. But since $\pi \circ c$ is an integral curve of $\tilde{\xi}^{h}$ these iterated derivatives exactly correspond to iterated covariant derivatives of $\mathrm{P}$ in direction $\xi$ evaluated at $\xi$. Thus, we obtain $\left(\nabla_{\xi} \ldots \nabla_{\xi} \mathbf{P}\right)(\xi)=0$ for any number of covariant derivatives. Using polarization, this implies that the symmetrization of $\left(\nabla_{\xi_{k}} \ldots \nabla_{\xi_{1}} \mathrm{P}\right)\left(\xi_{0}\right)$ over all $\xi_{i}$ vanishes at $x$.

To see that our condition fixes the infinite jet of the Weyl-structure suppose that $\hat{\sigma}$ is another normal Weyl structure with $\hat{\sigma}\left(u_{0}\right)=u$ and let $\Upsilon=$ $\left(\Upsilon_{1}, \ldots, \Upsilon_{k}\right)$ be the section of $\mathscr{A}_{1} \oplus \cdots \oplus \mathscr{A}_{k}$ describing the change from $\sigma$ to $\hat{\sigma}$. We want to show that the infinite jet of $\Upsilon$ vanishes at $x=p(u)$. Since both Weyl-structures map $u_{0}$ to $u$, we must have $\Upsilon(x)=0$. Next, we know that $\mathrm{P}(x)=\hat{\mathrm{P}}(x)=0$. Since all $\Upsilon_{i}$ vanish in $x$, formula (2) from Proposition 3.4 immediately shows that this implies $\nabla \Upsilon_{i}(x)=0$ for all $i=1, \ldots, k$, so $\nabla \Upsilon(x)=0$. Now, $\hat{\mathrm{P}}=0$ and $\Upsilon=0$. On one hand, it follows that $\hat{\nabla} \mathrm{P}(x)=\nabla \mathrm{P}(x)$ and on the other hand that $\left(\nabla_{\eta} \hat{\mathrm{P}}\right)(\xi)(x)=\nabla_{\eta}(\hat{\mathrm{P}}(\xi))(x)$. But hitting formula (2) from Proposition 3.4 with $\nabla_{\eta}$ and symmetrizing over $\xi$ and $\eta$, we always get terms involving some $\Upsilon_{i}$ or $\nabla_{\eta} \Upsilon_{i}$ or $\nabla_{\eta} \mathrm{P}(\xi)$ which all vanish at $x$, except for one term in the very last line, in which we get a second covariant derivative of some $\Upsilon_{i}$. So we see that the symmetrizations of $\hat{\nabla}_{\eta} \hat{\mathrm{P}}(\xi)$ and $\nabla_{\eta} \nabla_{\xi} \Upsilon$ coincide. Thus vanishing of the symmetrization of the first covariant derivative implies that the symmetrized second derivative of $\Upsilon$ is zero, and thus the two-jet of $\Upsilon$ at $x$ must be zero. Iteratively, one similarly sees that in the expression of an symmetrized iterated covariant derivative of $\mathbf{P}$ we always 
get terms involving symmetrized iterated covariant derivatives of $\Upsilon_{i}$ 's or P's except for one term coming from the very last line of the transformation formula. As above, one then concludes that vanishing of the symmetrization of the $k$-fold covariant derivative of $\hat{\mathrm{P}}$ is equivalent to vanishing of the symmetrization of the $(k+1)$-fold covariant derivative of $\Upsilon$ and thus to the $k+1$-jet of $\Upsilon$ in $x$ being trivial.

\section{Characterization of Weyl-structures}

In the last section, we started with a Weyl-structure for a parabolic geometry $(\mathscr{G} \rightarrow M, \omega)$ and we constructed several underlying objects on the manifold $M$, see Figure 1 for an illustration. Now we are going to characterize when general objects of that type actually come from a Weyl structure. In the final stage, this will mean explicit conditions relating the soldering form, linear connection and its torsion and curvature, together with a procedure building the corresponding Rho-tensors. This is quite simple for irreducible geometries, where the soldering form is fixed, and the whole condition prescribes uniquely the torsion of a $G_{0}$-connection. The Rho-tensor is then given by a simple formula in terms of the curvature, see Example 4.7 below. Of course, the same story gets much more complicated for the general $|k|$-graded case. The main step is done in Theorem 4.1 and then a detailed analysis of the curvature fulfills our goal.

Throughout this section we restrict to the case of regular parabolic geometries associated to a $|k|$-graded semisimple Lie algebra $\mathfrak{g}$ such that $H^{1}\left(\mathfrak{g}_{-}, \mathfrak{g}\right)$ is concentrated in homogeneous degrees $\leq 0$, i.e. such that none of the simple $\left|k_{i}\right|$-graded ideals is of one of the two types mentioned in Theorem 2.2. In the case that such ideals are present, a similar characterization is possible, but the conditions are more complicated to formulate.

\subsection{Definition}

Let $p_{0}: \mathscr{G}_{0} \rightarrow M$ be a regular infinitesimal flag structure, see 2.6. A Weylform for $M$ is a one-form $\tau \in \Omega^{1}\left(\mathscr{G}_{0}, \mathfrak{g}\right)$ which is $G_{0}$-equivariant, i.e. $\left(r^{g}\right)^{*} \tau=$ $\operatorname{Ad}\left(g^{-1}\right) \circ \tau$ for all $g \in G_{0}$, reproduces the generators of fundamental vector fields, i.e. $\tau\left(\zeta_{A}\right)=A$ for all $A \in \mathrm{g}_{0}$ and has the property that for each $i<0$ the restriction of $\tau_{i}$ to $T^{i} \mathscr{G}_{0}$ coincides with the $\mathrm{g}_{i}$-component of the frame form of degree one on $\mathscr{G}_{0}$ induced by the regular infinitesimal flag structure, see 2.6 and 3.4.

By 3.2 and 3.3, for any Weyl-structure $\sigma: \mathscr{G}_{0} \rightarrow \mathscr{G}$, the pullback $\sigma^{*} \omega$ is a Weyl-form for $M$. As in 3.3, the condition of the restriction of $\tau_{i}$ to $T^{i} \mathscr{G}_{0}$, $i<0$, means on $M$ exactly that the restriction of $\tau_{i}$ to $T^{i} M$ coincides with the canonical projection $T^{i} M \rightarrow \mathscr{A}_{i}$. In particular, this implies that $\tau_{-}=$ 
$\tau_{-k} \oplus \cdots \oplus \tau_{-1}$ induces a linear isomorphism $T_{u} \mathscr{G}_{0} / V_{u} \mathscr{G}_{0} \cong \mathfrak{g}_{-}$, and thus $\tau_{\leq 0}$ is a Cartan connection on $\mathscr{G}_{0}$. Completely parallel to the development in 3.2-3.4 we can equivalently interpret a Weyl-form for $M$ as a one form $\tau_{-} \in \Omega^{1}\left(M, \mathscr{A}_{-k} \oplus \cdots \oplus \mathscr{A}_{-1}\right)$ inducing an isomorphism between $T M$ and its associated graded bundle, plus a principal connection $\tau_{0} \in \Omega^{1}\left(\mathscr{G}_{0}, \mathfrak{g}_{0}\right)$ on $\mathscr{G}_{0}$, plus a Rho-tensor $\mathrm{P}=\mathrm{P}^{\tau} \in \Omega^{1}\left(M, \mathscr{A}_{1} \oplus \cdots \oplus \mathscr{A}_{k}\right)$, so a Weyl-form essentially consists of objects living on $M$.

\subsection{Weyl-curvature}

Next, for a Weyl-form $\tau$ for $M$, we define the Weyl-curvature $W$ of $\tau$. As a $\mathrm{g}$-valued two form on $\mathscr{G}_{0}$, it is defined by

$$
W(\xi, \eta)=d \tau(\xi, \eta)+[\tau(\xi), \tau(\eta)] .
$$

From the fact that $\tau$ is $G_{0}$-equivariant and reproduces the generators of fundamental vector fields, one immediately concludes that $W$ is horizontal and $G_{0}$-equivariant, so it descends to an $\mathscr{A}$-valued two form on $M$. Taking into account the identification of $T M$ with $\mathscr{A}_{-}$, we can also view $W$ as a section of $L\left(\Lambda^{2} \mathscr{A}_{-}, \mathscr{A}\right)$.

Finally note that any section $\Phi$ of $L\left(\Lambda^{2} \mathscr{A}_{-}, \mathscr{A}\right)$ can be split according to homogeneous degrees. We denote by $\Phi^{(\ell)}$ the homogeneous part of degree $\ell$, i.e. $\Phi^{(\ell)}(\xi, \eta) \in \mathscr{A}_{i+j+\ell}$ for sections $\xi$ of $\mathscr{A}_{i}$ and $\eta$ of $\mathscr{A}_{j}$ with $i, j<0$.

Lemma. Let $p_{0}: \mathscr{G}_{0} \rightarrow M$ be a regular infinitesimal flag structure. Then any Weyl-form $\tau \in \Omega^{1}\left(\mathscr{G}_{0}, \mathfrak{g}\right)$ has the property that $W^{(\ell)}=0$ for all $\ell \leq 0$.

Proof. Consider $\xi \in \Gamma\left(\mathscr{A}_{i}\right)$ and $\eta \in \Gamma\left(\mathscr{A}_{j}\right)$, for $i, j<0$. Then $\tau_{n}(\xi)=0$ for $n<i$ and $\tau_{m}(\eta)=0$ for $m<j$, so for $\ell<0$ if $m+n=i+j+\ell$ then $\left[\tau_{n}(\xi), \tau_{m}(\eta)\right]=0$. Thus, in this case, the definition of $W^{(\ell)}(\xi, \eta)$ can be rewritten as $W^{(\ell)}(\xi, \eta)=d \tau_{i+j+\ell}(\xi, \eta)=-\tau_{i+j+\ell}([\xi, \eta])$. By definition of a Weyl-form, $W^{(\ell)}(\xi, \eta)$ thus equals the class of the bracket $-[\xi, \eta]$ in $T^{i+j+\ell} M / T^{i+j+\ell+1} M$. But according to 2.6, we in particular know that the bracket of any section of $T^{i} M$ with a section of $T^{j} M$ lies in $T^{i+j} M$, so since $\ell<0$, we must have $W^{(\ell)}=0$.

Next, for $\ell=0$, we can write

$$
W^{(0)}(\xi, \eta)=d \tau_{i+j}(\xi, \eta)+\{\xi, \eta\}=-\tau_{i+j}([\xi, \eta])+\{\xi, \eta\} .
$$

Again, $\tau_{i+j}([\xi, \eta])$ is just the class of the bracket in $T^{i+j} M / T^{i+j+1} M$ and so the vanishing of $W^{(0)}$ is just the remaining part of the definition of regular infinitesimal flag structures, see 2.6. 


\subsection{Definition}

Let $p_{0}: \mathscr{G}_{0} \rightarrow M$ be a regular infinitesimal flag structure. Then a Weylform $\tau \in \Omega^{1}\left(\mathscr{G}_{0}, \mathfrak{g}\right)$ is called normal if and only if its Weyl-curvature $W \in$ $\Gamma\left(L\left(\Lambda^{2} \mathscr{A}_{-}, \mathscr{A}\right)\right)$ satisfies $\partial^{*}(W)=0$, where $\left.\partial^{*}: L\left(\Lambda^{2} \mathscr{A}_{-}, \mathscr{A}\right)\right) \rightarrow L\left(\mathscr{A}_{-}, \mathscr{A}\right)$ is the bundle map induced by the Lie algebra codifferential, see 2.11 .

Theorem 4.1. Let ( $p: \mathscr{G} \rightarrow M, \omega)$ be a regular parabolic geometry and let $p_{0}: \mathscr{G}_{0} \rightarrow M$ be the underlying regular infinitesimal flag structure. Then a Weyl-form $\tau \in \Omega^{1}\left(\mathscr{G}_{0}, \mathfrak{g}\right)$ for $M$ is coming from some Weyl-structure $\sigma: \mathscr{G}_{0} \rightarrow \mathscr{G}$, i.e. $\tau=\sigma^{*} \omega$, if and only if $\tau$ is normal.

Proof. First we show that for any Weyl-structure $\sigma: \mathscr{G}_{0} \rightarrow \mathscr{G}$ the Weylform $\sigma^{*} \omega$ is normal. By the definition in 4.2 the Weyl-curvature $W$ is a $g$-valued two-form on $\mathscr{G}_{0}$, given by

$$
W(\xi, \eta)=d \sigma^{*} \omega(\xi, \eta)+\left[\sigma^{*} \omega(\xi), \sigma^{*} \omega(\eta)\right]=\sigma^{*}\left(d \omega+\frac{1}{2}[\omega, \omega]\right)(\xi, \eta) .
$$

Thus, $W$ is simply the pullback along $\sigma$ of the curvature of the Cartan connection $\omega$ on $\mathscr{G}_{0}$. By definition of a normal parabolic geometry, this curvature is $\partial^{*}$-closed, so the same is true for $W$.

Now, let us assume that we have given an arbitrary normal Weyl-form $\tau \in \Omega^{1}\left(\mathscr{G}_{0}, \mathfrak{g}\right)$. Moreover, let us choose any bundle $\mathscr{L}^{\lambda}$ of scales for the parabolic geometry in question. Since $\tau_{0}$ is a principal connection on $\mathscr{G}_{0}$, it induces a principal connection on $\mathscr{L}^{\lambda}$, which by Theorem 3.8 in turn gives rise to a unique Weyl-structure $\sigma: \mathscr{G}_{0} \rightarrow \mathscr{G}$ such that the connection on $\mathscr{L}^{\lambda}$ induced by $\sigma^{*} \omega_{0}$ coincides with the connection induced by $\tau_{0}$. We claim that $\tau=\sigma^{*} \omega$, which will conclude the proof.

Consider the difference $\tau-\sigma^{*} \omega \in \Omega^{1}\left(\mathscr{G}_{0}, \mathfrak{g}\right)$. For $i<0$, we know from our assumptions that both $\tau_{i}$ and $\sigma^{*} \omega_{i}$ coincide on $T^{i} \mathscr{G}_{0}$ with the frame form of degree one. In particular, the difference $\tau_{i}-\sigma^{*} \omega_{i}$ vanishes on $T^{i} \mathscr{G}_{0}$ for all $i<0$. Since $T^{0} \mathscr{G}_{0}$ is just the vertical bundle of $\mathscr{G}_{0}$ and since both $\tau_{0}$ and $\sigma^{*} \omega_{0}$ are principal connections on $\mathscr{G}_{0}$, we see that $\tau_{0}-\sigma^{*} \omega_{0}$ vanishes on $T^{0} \mathscr{G}_{0}$, too. Finally, if we put $T^{i} \mathscr{G}_{0}$ to be the zero section for $i>0$, then $\tau_{i}-\sigma^{*} \omega_{i}$ vanishes on $T^{i} \mathscr{G}_{0}$ for all $i=-k, \ldots, k$. Let us inductively assume that $\tau_{i}-\sigma^{*} \omega_{i}$ vanishes on $T^{i-n+1} \mathscr{G}_{0}$ for all $i$ and some $n$.

Then consider the restriction of $\tau_{i}-\sigma^{*} \omega_{i}$ to $T^{i-n} \mathscr{G}_{0}$, which can be viewed as a map $T^{i-n} \mathscr{G}_{0} / T^{i-n+1} \mathscr{G}_{0} \rightarrow \mathrm{g}_{i}$. For each $i$ such that $i-n \leq 0$, the forms $\tau_{i-n}$ and $\sigma^{*} \omega_{i-n}$ coincide on $T^{i-n} \mathscr{G}_{0}$ and induce an isomorphism $T^{i-n} \mathscr{G}_{0} / T^{i-n+1} \mathscr{G}_{0}$ $\rightarrow \mathscr{G}_{0} \times \mathfrak{g}_{i-n}$. Consequently, we get a unique map $\Phi: \mathscr{G}_{0} \rightarrow L\left(\mathfrak{g}_{-}, \mathfrak{g}\right)$ which has values in maps homogeneous of degree $n$, such that $\left(\tau_{i}-\sigma^{*} \omega_{i}\right)(\xi)=$ $\Phi\left(\tau_{i-n}(\xi)\right)$ for all $\xi \in T^{i-n} \mathscr{G}_{0}$. 
Next, let $W^{(n)}$ be the homogeneous component of degree $n$ of the Weylcurvature of $\tau$ viewed as a function $\mathscr{G}_{0} \rightarrow L\left(\Lambda^{2} \mathfrak{g}_{-}, \mathfrak{g}\right.$ ) (having values in the maps homogeneous of degree $n$ ), and let $\tilde{W}^{(n)}$ be the corresponding object for $\sigma^{*} \omega$. We claim that for all $X, Y \in \mathfrak{g}_{-}$

$$
\begin{aligned}
\tilde{W}^{(n)}(X, Y) & =W^{(n)}(X, Y)-[X, \Phi(Y)]+[Y, \Phi(X)]+\Phi([X, Y]) \\
& =W^{(n)}(X, Y)-(\partial \circ \Phi)(X, Y)
\end{aligned}
$$

Let us postpone the proof of this claim and assume it is true for a while. Since both $W^{(n)}$ and $\tilde{W}^{(n)}$ are $\partial^{*}$-closed, this implies $\partial^{*} \circ \partial \circ \Phi=0$, which implies $\partial \circ \Phi=0$ since $\partial$ and $\partial^{*}$ are adjoint, see 2.4. Since $H^{1}\left(\mathfrak{g}_{-}, \mathfrak{g}\right)$ is concentrated in non-positive degrees of homogeneity, this implies $\Phi=0$ for $n>k$ and $\Phi(X)=[Z, X]$ for some smooth $Z: \mathscr{G}_{0} \rightarrow \mathfrak{g}_{n}$ for $n \leq k$. But in the latter case, the proof of Theorem 3.8(1) shows that since $\tau_{0}$ and $\sigma^{*} \omega_{0}$ induce the same principal connection on $\mathscr{L}^{\lambda}$, we must have $Z=0$, and thus $\Phi=0$. Hence, $\tau_{i}$ and $\sigma^{*} \omega_{i}$ coincide on $T^{i-n} \mathscr{G}_{0}$ for all $i<n$, for $i=n$ this follows since $n>0$ and thus both $\tau_{n}$ and $\sigma^{*} \omega_{n}$ are horizontal, while for $i>n$ it is trivially satisfied. Thus the result follows by induction.

So we are left with the proof of (1) only. Let us fix $X \in \mathfrak{g}_{i}, Y \in \mathfrak{g}_{j}, i, j<0$. By definition,

$$
W^{(n)}(u)(X, Y)=d \tau_{i+j+n}\left(\tau_{\leq 0}^{-1}(X), \tau_{\leq 0}^{-1}(Y)\right)+\left[\tau\left(\tau_{\leq 0}^{-1}(X)\right), \tau\left(\tau_{\leq 0}^{-1}(Y)\right)\right]_{i+j+n},
$$

where the index in the bracket means that we just have to take the component in $g_{i+j+n}$. For $\tilde{W}^{(n)}$ we get the analogous formula with all $\tau$ 's replaced by $\sigma^{*} \omega$.

Next, observe that both $\tau_{\leq 0}^{-1}(X)$ and $\sigma^{*} \omega_{\leq 0}^{-1}(X)$ lie in $T^{i} \mathscr{G}_{0} \subset T^{i+j} \mathscr{G}_{0}$ and similarly for $Y$. From above, we know that $\sigma^{*} \omega_{i+j+n}(\xi)=\tau_{i+j+n}(\xi)-$ $\Phi\left(\tau_{i+j}(\xi)\right)$ for all $\xi$ in $T^{i+j} \mathscr{G}_{0}$. Taking the exterior derivative of this equation and keeping in mind that $\tau_{i+j}$ vanishes on $T^{i} \mathscr{G}_{0}$ and $T^{j} \mathscr{G}_{0}$, we see that for $\xi \in T^{i} \mathscr{G}_{0}$ and $\eta \in T^{j} \mathscr{G}_{0}$ we get

$$
d \sigma^{*} \omega_{i+j+n}(\xi, \eta)=d \tau_{i+j+n}(\xi, \eta)-\Phi\left(d \tau_{i+j}(\xi, \eta)\right) .
$$

Since $W^{(0)}=0$, the second term (including the - sign) can be rewritten as $\Phi\left(\left[\tau_{i}(\xi), \tau_{j}(\eta)\right]\right)$, and we may as well replace $\tau$ by $\sigma^{*} \omega$ in this expression. Thus, we see that

$$
\begin{aligned}
\tilde{W}^{(n)}(X, Y)=d & \tau_{i+j+n}\left(\sigma^{*} \omega_{\leq 0}^{-1}(X), \sigma^{*} \omega_{\leq 0}^{-1}(Y)\right)+\Phi([X, Y]) \\
+ & {\left[\sigma^{*} \omega\left(\sigma^{*} \omega_{\leq 0}^{-1}(X)\right), \sigma^{*} \omega\left(\sigma^{*} \omega_{\leq 0}^{-1}(Y)\right)\right]_{i+j+n} . }
\end{aligned}
$$

Now we have to distinguish a few cases: Let us first assume that $i+n>0$. Then $\sigma^{*} \omega_{\leq 0}^{-1}(X)=\tau_{\leq 0}^{-1}(X)$, and $\sigma^{*} \omega\left(\sigma^{*} \omega_{\leq 0}^{-1}(X)\right)=\tau\left(\tau_{\leq 0}^{-1}(X)\right)-\Phi(X)$, and 
$\Phi(X) \in \mathfrak{g}_{i+n} \subset \mathfrak{p}_{+}$. In particular, this implies that

$$
\begin{aligned}
{\left[\sigma^{*} \omega\left(\sigma^{*} \omega_{\leq 0}^{-1}(X)\right), \sigma^{*} \omega\left(\sigma^{*} \omega_{\leq 0}^{-1}(Y)\right)\right]_{i+j+n} } \\
=\left[\tau\left(\tau_{\leq 0}^{-1}(X)\right), \sigma^{*} \omega\left(\sigma^{*} \omega_{\leq 0}^{-1}(Y)\right)\right]_{i+j+n}-[\Phi(X), Y] .
\end{aligned}
$$

Secondly, if $i+n=0$ then $\Phi(X) \in \mathfrak{g}_{0}$, and thus $\sigma^{*} \omega_{\leq 0}^{-1}(X)=\tau_{\leq 0}^{-1}(X)+\zeta_{\Phi(X)}$. The infinitesimal version of equivariance of $\tau_{i+j+n}$ then implies that

$$
\begin{aligned}
& d \tau_{i+j+n}\left(\sigma^{*} \omega_{\leq 0}^{-1}(X), \sigma^{*} \omega_{\leq 0}^{-1}(Y)\right) \\
& \quad=d \tau_{i+j+n}\left(\tau_{\leq 0}^{-1}(X), \sigma^{*} \omega_{\leq 0}^{-1}(Y)\right)-[\Phi(X), Y],
\end{aligned}
$$

since $i+j+n=j$ in this case. On the other hand both $\sigma^{*} \omega\left(\sigma^{*} \omega_{\leq 0}^{-1}(X)\right)$ and $\tau\left(\tau_{\leq 0}^{-1}(X)\right)$ in this case are congruent to $X$ modulo $\mathfrak{p}_{+}$, so

$$
\begin{aligned}
{\left[\sigma^{*} \omega\left(\sigma^{*} \omega_{\leq 0}^{-1}(X)\right), \sigma^{*} \omega\left(\sigma^{*} \omega_{\leq 0}^{-1}(Y)\right)\right]_{i+j+n} } & \\
= & {\left[\tau\left(\tau_{\leq 0}^{-1}(X)\right), \sigma^{*} \omega\left(\sigma^{*} \omega_{\leq 0}^{-1}(Y)\right)\right]_{i+j+n} . }
\end{aligned}
$$

Finally, suppose that $i+n<0$, so $\Phi(X) \in \mathfrak{g}_{i+n} \subset \mathfrak{g}_{-}$. Then $\sigma^{*} \omega_{\leq 0}^{-1}(X)$ is congruent to $\tau_{\leq 0}^{-1}(X+\Phi(X))$ modulo $T^{i+n+1} \mathscr{G}_{0}$. Since the bracket of a section of this subbundle with a section of $T^{j} \mathscr{G}_{0}$ is a section of $T^{i+j+n+1} \mathscr{G}_{0}$ and $\tau_{i+j+n}$ vanishes on the latter subbundle, we conclude that

$$
\begin{aligned}
d \tau_{i+j+n}\left(\sigma^{*} \omega_{\leq 0}^{-1}(X), \sigma^{*} \omega_{\leq 0}^{-1}(Y)\right)= & d \tau_{i+j+n}\left(\tau_{\leq 0}^{-1}(X), \sigma^{*} \omega_{\leq 0}^{-1}(Y)\right) \\
& +d \tau_{i+j+n}\left(\tau_{\leq 0}^{-1}(\Phi(X)), \sigma^{*} \omega_{\leq 0}^{-1}(Y)\right) .
\end{aligned}
$$

Since $W^{(0)}=0$, the last term can be rewritten as $-[\Phi(X), Y]$. As above, both $\sigma^{*} \omega\left(\sigma^{*} \omega_{\leq 0}^{-1}(X)\right)$ and $\tau\left(\tau_{\leq 0}^{-1}(X)\right)$ are congruent to $X$ modulo $\mathfrak{p}_{+}$, so again the bracket term makes no problem.

Hence we see, that in any case we get

$$
\begin{aligned}
\tilde{W}^{(n)}(X, Y) & =d \tau_{i+j+n}\left(\tau_{\leq 0}^{-1}(X), \sigma^{*} \omega_{\leq 0}^{-1}(Y)\right) \\
+ & {\left[\tau\left(\tau_{\leq 0}^{-1}(X)\right), \sigma^{*} \omega\left(\sigma^{*} \omega_{\leq 0}^{-1}(Y)\right)\right]_{i+j+n}-[\Phi(X), Y]+\Phi([X, Y]) . }
\end{aligned}
$$

Doing the same changes to $Y$ instead of $X$ we obtain the required equality (1), and the whole proof of the theorem is finished.

\subsection{Remark}

If one does not assume that $H^{1}\left(\mathfrak{g}_{-}, \mathfrak{g}\right)$ is concentrated in non-positive degrees, i.e. if one allows $\mathfrak{g}$ to contain one of the two simple factors mentioned in Theorem 2.2, then $H^{1}\left(\mathfrak{g}_{-}, \mathfrak{g}\right)$ is concentrated in homogeneous degrees less or 
equal to one. Thus, the above proof shows that $\tau=\sigma^{*} \omega$ if $\tau$ is normal and has the property that the restrictions of $\tau_{i}$ and $\sigma^{*} \omega_{i}$ to $T^{i-1} \mathscr{G}_{0}$ coincide for all $i$. This condition is then fairly simple to interpret for any concrete choice of such structure.

\section{5 .}

In the proof of Theorem 4.1, we observed that for a Weyl-structure $\sigma: \mathscr{G}_{0} \rightarrow \mathscr{G}$ the Weyl-curvature $W$ of the Weyl-form $\sigma^{*} \omega$ is exactly the pullback along $\sigma$ of the curvature $\kappa$ of the normal Cartan connection $\omega$ on $\mathscr{G}$. This allows us to compute the change of the Weyl-curvature under a change of the Weylstructure. Suppose that $\hat{\sigma}$ is another Weyl-structure and $\Upsilon=\left(\Upsilon_{1}, \ldots, \Upsilon_{k}\right)$ is the smooth section of $\mathscr{A}_{1} \oplus \cdots \oplus \mathscr{A}_{k}$ describing the change from $\sigma$ to $\hat{\sigma}$, see Proposition 3.1, i.e.

$$
\hat{\sigma}(u)=\sigma(u) \exp \left(\Upsilon_{1}(u)\right) \ldots \exp \left(\Upsilon_{k}(u)\right) .
$$

Equivariance of the Cartan connection $\omega$ immediately implies that the curvature $\kappa$ is equivariant, i.e. viewing $\kappa$ as a two form on $\mathscr{G}$ with values in $\mathfrak{g}$, we have $\kappa(v \cdot g)\left(T r^{g} \cdot \xi, T r^{g} \cdot \eta\right)=\operatorname{Ad}\left(g^{-1}\right)(\kappa(v)(\xi, \eta))$ for $g \in P$ and $\xi, \eta \in T_{v} \mathscr{G}$. Putting $v=\sigma(u)$ and $g=\exp \left(\Upsilon_{1}(u)\right) \ldots \exp \left(\Upsilon_{k}(u)\right)$, we see from the proof of Proposition 3.4 that for $\xi \in T_{u} \mathscr{G}_{0}$ the element $T_{u} \hat{\sigma} \cdot \xi$ is congruent to $\operatorname{Tr}^{g} T_{u} \sigma \cdot \xi$ modulo vertical elements, which are killed by the curvature anyhow. Thus, viewing $W$ and $\hat{W}$ as $g$-valued two forms on $\mathscr{G}_{0}$, we get $\hat{W}(\xi, \eta)=\operatorname{Ad}\left(g^{-1}\right)(W(\xi, \eta))$. Moreover, to get the interpretation of our two Weyl curvatures $W$ and $\hat{W}$ as $\mathscr{A}$-valued two forms on $M$, we just have to apply the above definition to lifts of vector fields on $M$, and the result is independent of the choice of the lifts since $W$ is horizontal. Keeping in mind that the Lie-bracket in $\mathrm{g}$ corresponds to the algebraic bracket of sections of $\mathscr{A}$ and expanding the exponentials in $\operatorname{Ad}\left(g^{-1}\right)$ as in the proof of Proposition 3.4 we arrive (with notation as in 3.4) at

$$
\hat{W}_{i}(\xi, \eta)=\sum_{\|\underline{j}\|+\ell=i} \frac{(-1)^{\underline{j}}}{\underline{j} !} \operatorname{ad}\left(\Upsilon_{k}\right)^{j_{k}} \circ \cdots \circ \operatorname{ad}\left(\Upsilon_{1}\right)^{j_{1}}\left(W_{\ell}(\xi, \eta)\right) .
$$

From this formula, one can also derive a formula describing the change of $W$ viewed as a section of $L\left(\Lambda^{2} \mathscr{A}_{-}, \mathscr{A}\right)$ taking into account the change of the identification of $T M$ with $\mathscr{A}_{-}$described by (1) in Proposition 3.4, and thus a formula for the change of the individual homogeneous components $W^{(\ell)}$. The only point that is important for us here is that the homogeneous component $W^{(1)}$ of degree one is actually independent of $\sigma$. This can be immediately verified from the above formula, taking into account that $W^{(\ell)}=0$ for all $\ell \leq 0$. 


\subsection{Remark}

The results obtained so far in principle allow to give a description of the Cartan bundle and the Cartan connection completely in terms of data on the manifold $M$. More precisely, if we start from a regular infinitesimal flag structure underlying some parabolic geometry, then we may proceed as follows: Choose a scaling element $E_{\lambda} \in z\left(\mathrm{~g}_{0}\right)$, and consider the corresponding homomorphism $\lambda: G_{0} \rightarrow \mathrm{R}^{+}$described in the proof of Proposition 3.2. Then form $\mathscr{L}_{\lambda}=\mathscr{G}_{0} \times_{G_{0}} \mathrm{R}^{+}$. From Theorem 3.8(2) we then know that the Cartan bundle $\mathscr{G}$ is just the pullback of the bundle of principal connections on $\mathscr{L}_{\lambda}$, and we have a description of the principal action. Moreover, a choice of a principal connection on $\mathscr{L}^{\lambda}$ is just the choice of a global section of the bundle of connections, so its pullback is a smooth $G_{0}$-equivariant section $\sigma: \mathscr{G}_{0} \rightarrow \mathscr{G}$. Any Cartan connection $\omega$ on $\mathscr{G}$ is uniquely determined by its pullback $\sigma^{*} \omega$ by equivariance. Thus, describing the canonical normal Cartan connection on $\mathscr{G}$ is equivalent to finding a normal Weyl-form on $\mathscr{G}_{0}$ which induces a given connection on $\mathscr{L}^{\lambda}$.

\subsection{Example}

Let us look more closely at the irreducible parabolic geometries. Here the regular infinitesimal flag structures are just $G_{0}$-structures on $M$ in the sense of classical G-structures. The Weyl forms are $\tau=\tau_{-1}+\tau_{0}+\tau_{1}$ where $\tau_{-1}$ : $T \mathscr{G}_{0} \rightarrow \mathfrak{g}_{-1}$ is the fixed soldering form for $M, \tau_{0}$ is any linear connection on $M$ belonging to the fixed $G_{0}$-structure and $\tau_{1}$ is any one-form in $\Omega^{1}\left(M ; T^{*} M\right)$. Now,

$$
W_{-1}=d \tau_{-1}+\left[\tau_{-1}, \tau_{0}\right],
$$

i.e. the torsion of the connection $\tau_{0}$. The individual components of $W$ have homogeneities one, two, and three and so they have to be $\partial^{*}$-closed separately. The condition $\partial^{*} W_{-1}=0$ means that the torsion of $\tau_{0}$ is harmonic and this is the part of $W$ independent of the choice of the Weyl-structure. Next,

$$
W_{0}=d \tau_{0}+\frac{1}{2}\left[\tau_{0}, \tau_{0}\right]+\left[\tau_{-1}, \tau_{1}\right]
$$

which is the curvature $R$ of the connection $\tau_{0}$ plus some additional term. The co-closedness of $W_{0}$ imposes a condition on the choice of $\tau_{1}$, while $\partial^{*} W_{1}$ always vanishes since its values are in the trivial vector space.

We shall see later that the resulting system of equations for the tensor $\tau_{1}$ is always solvable, except for the projective structures (where the first cohomology is concentrated in degree one). Moreover, we shall prove an explicit algebraic formula for the necessary choice for the Rho-tensor: $\tau_{1}=\square^{-1} \partial^{*} R$. Expanding this formula in the case of the conformal (pseudo) Riemannian geometry, we obtain the well known Rho-tensor used heavily by many authors since the 
beginning of the 20th century, while $d \tau_{1}$ happens to be exactly another well known tensor, the Cotton-York tensor.

As mentioned above, this computation may be understood as an alternative for the explicit construction of the canonical Cartan connection for all irreducible parabolic geometries.

\subsection{Total curvature}

The explicit construction of a normal Weyl-form depends a lot on the structure in question, a detailed treatment in the case of partially integrable almost CRstructures of hypersurface type will appear in [6]. Here we just describe the basic ingredient of this procedure. The upshot of this is that the condition on a Weyl-form $\tau$ being normal can be step by step reduced to a condition on $\tau_{\leq 0}$ only, at the same time computing step by step the components of the Rho-tensor $\mathbf{P}=\tau_{+}$.

The first step in this direction is to replace the Weyl curvature of a Weyl-form $\tau$ by 2 -forms defined by splitting the structure equations for $\tau$. The curvature of the Cartan connection $\tau_{\leq 0}$ is the 2 -form $K_{\leq 0} \in \Omega^{2}\left(\mathscr{G}_{0}, \mathfrak{g}_{-}\right)$given by

$$
K_{\leq 0}(\xi, \eta)=d \tau_{\leq 0}(\xi, \eta)+\left[\tau_{\leq 0}(\xi), \tau_{\leq 0}(\eta)\right] .
$$

On the other hand, we define the 2 -form $K_{+} \in \Omega^{2}\left(\mathscr{G}_{0}, \mathfrak{p}_{+}\right)$by

$$
K_{+}(\xi, \eta)=d \tau_{+}(\xi, \eta)+\left[\tau_{+}(\xi), \tau_{+}(\eta)\right] .
$$

Motivated by conformal geometry, we call $K_{+}$the Cotton-York-tensor associated to the Weyl-form $\tau$. We write $K=K_{\leq 0}+K_{+}$and we call it the (total) curvature of $\tau$. Since $\tau_{\leq 0}$ is a Cartan connection, it is well known that its curvature is horizontal and $G_{0}$-equivariant, so it can be viewed as a two form on $M$, with values in the bundle $\mathscr{A}_{-k} \oplus \cdots \oplus \mathscr{A}_{0}$. On the other hand, since $\tau_{+}$is by assumption $G_{0}$-equivariant and horizontal, the part $K_{+}$descends to $M$, too. Finally, taking into account the isomorphism $T M \cong \mathscr{A}_{-}=\mathscr{A}_{-k} \oplus \cdots \oplus \mathscr{A}_{-1}$, we can finally view $K$ as a smooth section of the bundle $L\left(\Lambda^{2} \mathscr{A}_{-}, \mathscr{A}\right)$ over $M$.

The reason for introducing this curvature is that it is more closely related to usual invariants of the Weyl-form than the Weyl-curvature, cf. Example 4.7. On the other hand, we shall see that there still is a simple relation between curvature and Weyl-curvature.

To get explicit expressions for the components of $K$, recall that the component $\tau_{0}$ of any Weyl-form $\tau$ is a principal connection on $\mathscr{G}_{0}$, and thus induces a linear connection $\nabla$ on each of the bundles $\mathscr{A}_{i}$. Let us also recall that $\tau_{i}$ are identified with forms $\Omega^{1}\left(M ; \mathscr{A}_{i}\right)$ for all negative $i$. 
Proposition 4.2. Let $p_{0}: \mathscr{G}_{0} \rightarrow M$ be a regular infinitesimal flag structure, let $\tau \in \Omega^{1}\left(\mathscr{G}_{0}, \mathfrak{g}\right)$ be a Weyl-form for $M$, and let $K$ be its total curvature, viewed as an $\mathscr{A}$-valued two form on $M$ with $\mathscr{A}_{\ell}$-component $K_{\ell}$. Then for all vector fields $\xi$ and $\eta$ on $M$ we have:

(1) $K_{\ell}(\xi, \eta)=\nabla_{\xi}\left(\tau_{\ell}(\eta)\right)-\nabla_{\eta}\left(\tau_{\ell}(\xi)\right)-\tau_{\ell}([\xi, \eta])+\sum_{\substack{i, j<0 \\ i+j=\ell}}\left\{\tau_{i}(\xi), \tau_{j}(\eta)\right\}$, for $\ell<0$.

(2) For $\zeta \in \mathscr{A}_{m}$ we get $\left\{K_{0}(\xi, \eta), \zeta\right\}=R_{m}(\xi, \eta)(\zeta)$, where $R_{m}$ is the curvature of the linear connection $\nabla$ on $\mathscr{A}_{m}$.

Moreover, if we view $K$ as a section of $L\left(\Lambda^{2} \mathscr{A}_{-}, \mathscr{A}\right)$ and consider $\ell>0$, then the homogeneous component $K^{(\ell)}$ of $K$ depends only on the restrictions of $\tau_{i}$ to $T^{i-\ell \mathscr{G}_{0}}$ for all $i \leq 0$ and on the restrictions of $\tau_{i}$ to $T^{i-\ell+1} \mathscr{G}_{0}$ for $i>0$.

Proof. By definition, for $\ell<0$ the function $\mathscr{G}_{0} \rightarrow \mathfrak{g}_{\ell}$ corresponding to $K_{\ell}(\xi, \eta)$ is given by

$$
\begin{aligned}
& d \tau_{\ell}\left(\xi^{h}, \eta^{h}\right)+\sum_{i, j \leq 0, i+j=\ell}\left[\tau_{i}\left(\xi^{h}\right), \tau^{j}\left(\eta^{h}\right)\right] \\
& \quad=\xi^{h} \cdot \tau_{\ell}\left(\eta^{h}\right)-\eta^{h} \cdot \tau_{\ell}\left(\xi^{h}\right)-\tau_{\ell}\left(\left[\xi^{h}, \eta^{h}\right]\right)+\sum_{i, j \leq 0, i+j=\ell}\left[\tau_{i}\left(\xi^{h}\right), \tau_{j}\left(\eta^{h}\right)\right],
\end{aligned}
$$

where the superscript $h$ denotes the horizontal lift with respect to the principal connection $\tau_{0}$. But now $\tau_{\ell}\left(\eta^{h}\right): \mathscr{G}_{0} \rightarrow \mathfrak{g}_{\ell}$ is exactly the smooth function corresponding to the section $\tau_{\ell}(\eta)$ of $\mathscr{A}_{\ell}$, so the function $\xi^{h} \cdot \tau_{\ell}\left(\eta^{h}\right)$ corresponds to $\nabla_{\xi}\left(\tau_{\ell}(\eta)\right)$ and similarly for the second term. On the other hand, [ $\left.\xi^{h}, \eta^{h}\right]$ is a lift of the vector field $[\xi, \eta]$, so since $\tau_{\ell}$ is horizontal for $\ell<0$, we see that the function $\tau_{\ell}\left(\left[\xi^{h}, \eta^{h}\right]\right)$ corresponds to the section $\tau_{\ell}([\xi, \eta])$ of $\mathscr{A}_{\ell}$. Finally, for the last sum one only has to take into account that $\tau_{0}$ vanishes on horizontal lifts and the bracket in $\mathrm{g}$ corresponds to the algebraic bracket on $\mathscr{A}$.

If $\ell=0$, the definition of $K_{0}$ reduces to $d \tau_{0}\left(\xi^{h}, \eta^{h}\right)$ and this exactly represents the curvature of the principal connection $\tau_{0}$, so the result follows immediately, taking into account that the action of $\mathrm{g}_{0}$ on $\mathfrak{g}_{m}$ is given by the Lie bracket in $\mathrm{g}$ and thus corresponds to the algebraic bracket $\mathscr{A}_{0} \times \mathscr{A}_{m} \rightarrow \mathscr{A}_{m}$.

To verify the statements about homogeneous degrees, take sections $\xi$ of $\mathscr{A}_{i}$ and $\eta$ of $\mathscr{A}_{j}$, and let $\tilde{\xi}$ be the (unique) section of $T^{i} M$ such that $\tau_{n}(\tilde{\xi})=0$ for all $i<n<0, \tau_{i}(\tilde{\xi})=\xi$, and similarly for $\tilde{\eta}$. Then for $\ell>0, K^{(\ell)}(\xi, \eta)=$ $K_{i+j+\ell}(\tilde{\xi}, \tilde{\eta})$. If $i+j+\ell<0$, then the above formula just gives us

$$
\delta_{i+\ell}^{0} \nabla_{\tilde{\xi}} \eta-\delta_{j+\ell}^{0} \nabla_{\tilde{\eta}} \xi-\tau_{i+j+\ell}([\tilde{\xi}, \tilde{\eta}]) .
$$

This is completely independent of the components $\tau_{n}$ for $n>0$. If we allow a change of $\tau$ without changing the restriction of $\tau_{n}$ to $T^{n-\ell}$ for all $n \leq 0$, then 
this means that $\tilde{\xi}$ is changed at most by a section of $T^{i+\ell+1} M$. In particular, if the first term in the above expression actually occurs, i.e. $i+\ell=0$ then $\tilde{\xi}$ is fixed, and moreover, since the restriction of $\tau_{0}$ to $T^{-\ell} \mathscr{G}_{0}=T^{i} \mathscr{G}_{0}$ is fixed, also the covariant derivative is fixed. Similarly one analyzes the second term. Finally, the last term depends only on the restriction of $\tau$ since the bracket of a section of $T^{i+\ell+1} M$ with a section of $T^{j} M$ is a section of $T^{i+j+\ell+1} M$ and this subbundle lies in the kernel of $\tau_{i+j+\ell}$.

If $i+j+\ell=0$, then $K^{(\ell)}(\xi, \eta)=d \tau_{0}\left((\tilde{\xi})^{h},(\tilde{\eta})^{h}\right)$, and as above, we see that $(\tilde{\xi})^{h}$ and $(\tilde{\eta})^{h}$ depend only on the appropriate restriction of $\tau$. Moreover, the bracket $\left[(\tilde{\xi})^{h},(\tilde{\eta})^{h}\right]$ by construction is a section of $T^{i+j} \mathscr{G}_{0}$, so the whole expression depends only on the restriction of $\tau_{0}$ to $T^{i+j} \mathscr{G}_{0}=T^{0-\ell} \mathscr{G}_{0}$.

Finally, we have to consider the case $i+j+\ell>0$, so we are dealing with a component of $K$ having values in $\mathscr{A}_{+}$. As before, one verifies that all extensions and horizontal lifts depend only on the appropriate restrictions of $\tau_{\leq 0}$, so what remains to be discussed is the dependence on $\mathbf{P}$. But viewing $\mathbf{P}$ as a section of $L\left(\mathscr{A}_{-}, \mathscr{A}_{+}\right)$, the statement to be proved reduces to the fact that a homogeneous component of $K$ depends only on homogeneous components of $\mathrm{P}$ of strictly smaller degree. But this is obvious from the definition of $K_{+}$.

\subsection{Remark}

The previous Proposition reveals that the $\mathscr{A}_{-}$-components of the total curvature give exactly the torsion of the linear connection $\tau_{0}$ corrected by the algebraic contribution of the Lie bracket in $\mathrm{g}_{-}$, while the component $K_{0}$ is just the standard curvature of $\tau_{0}$. For a normal Weyl form $\tau$ this means (using Proposition 4.3 below) that the torsion of $\tau_{0}$ has the algebraic bracket as its homogeneous component of degree zero, no components of negative degrees, and some positive degree components. The torsion component of degree one is an invariant of the parabolic structure in question.

The key point in the further analysis is that while the total curvature of a Weyl-form is much easier to relate to the underlying structure than its Weylcurvature, there is the quite simple relation between them described in the next Proposition.

Proposition 4.3. Let $\tau \in \Omega^{1}\left(\mathscr{G}_{0}, \mathfrak{g}\right)$ be a Weyl-form for $M$, let $\mathrm{P} \in$ $\Gamma\left(L\left(\mathscr{A}_{-}, \mathscr{A}_{+}\right)\right)$be its Rho-tensor, and let $K, W \in \Gamma\left(L\left(\Lambda^{2} \mathscr{A}_{-}, \mathscr{A}\right)\right)$ be its total curvature and its Weyl-curvature, respectively. Then

$$
W(\xi, \eta)=K(\xi, \eta)+\{\mathrm{P}(\xi), \eta\}-\{\mathrm{P}(\eta), \xi\} .
$$

In particular, $W^{(i)}=K^{(i)}$ for all $i \leq 1$.

Proof. Let $\xi$ be a section of $\mathscr{A}_{i}$ and $\eta$ be a section of $\mathscr{A}_{j}$, with $i, j<0$. To compute $W(\xi, \eta)$, we first have to view $\xi$ and $\eta$ as vector fields on $M$ via 
$\tau_{-}: T M \cong \mathscr{A}_{-}$. Then, by construction the section $W(\xi, \eta)$ of $\mathscr{A}$ corresponds to the function $\mathscr{G}_{0} \rightarrow \mathfrak{g}$ given by

$$
d \tau\left(\xi^{h}, \eta^{h}\right)+\left[\tau\left(\xi^{h}\right), \tau\left(\eta^{h}\right)\right],
$$

where the subscript $h$ denotes the horizontal lift with respect to the principal connection $\tau_{0}$. Thus, the $g_{0}$-components of $\tau\left(\xi^{h}\right)$ and $\tau\left(\eta^{h}\right)$ are automatically zero, so we may write

$$
\begin{aligned}
{\left[\tau\left(\xi^{h}\right), \tau\left(\eta^{h}\right)\right]=[} & {\left[\tau_{-}\left(\xi^{h}\right), \tau_{-}\left(\eta^{h}\right)\right]+\left[\tau_{+}\left(\xi^{h}\right), \tau_{-}\left(\eta^{h}\right)\right] } \\
& +\left[\tau_{-}\left(\xi^{h}\right), \tau_{+}\left(\eta^{h}\right)\right]+\left[\tau_{+}\left(\xi^{h}\right), \tau_{+}\left(\eta^{h}\right)\right] .
\end{aligned}
$$

On the other hand, from the definition of the curvature it is clear, that the section $K(\xi, \eta)$ corresponds to the function

$$
d \tau\left(\xi^{h}, \eta^{h}\right)+\left[\tau_{-}\left(\xi^{h}\right), \tau_{-}\left(\eta^{h}\right)\right]+\left[\tau_{+}\left(\xi^{h}\right), \tau_{+}\left(\eta^{h}\right)\right] .
$$

Now $\tau_{+}\left(\xi^{h}\right)$ is exactly the function corresponding to $P(\xi)$, while $\tau_{-}\left(\eta^{h}\right)$ is the function corresponding to $\eta$. (Actually, by construction $\tau_{-}(\eta)$ has values in $\mathfrak{g}_{j}$ only, but this is not important here.) Since the algebraic bracket $\{$,$\} is simply$ induced by the Lie bracket on $\mathfrak{g}$, the formula for $W(\xi, \eta)$ follows immediately.

To see the second statement, one just has to notice that the algebraic bracket is by definition homogeneous of degree zero, while all nonzero homogeneous components of $\mathrm{P}$ have degree at least two.

\subsection{Remark}

Note that the latter result, together with the formula (1) for the change of the Weyl-curvature of a Weyl-structure from 4.5 and the formula (2) for the change of the Rho-tensor from Proposition 3.4, gives us a formula for the change of the total curvature of a Weyl-structure under the change of the Weyl-structure.

\subsection{The construction of normal Weyl-forms}

Now we are ready to describe the procedure of step by step reducing the condition of normality of a Weyl-form $\tau \in \Omega^{1}\left(\mathscr{G}_{0}, \mathfrak{g}\right)$ to a condition on $\tau_{\leq 0}$ and at the same time computing step by step the Rho-tensor. From Proposition 4.3 we know that $W^{(1)}=K^{(1)}$ and from 4.5 we know that this is actually the same expression for any normal Weyl-form. Usually, this can be computed in advance, and thus gives us a condition on the restriction of $\tau_{i}$ to $T^{i-1} \mathscr{G}_{0}$ for $i \leq 0$. Next, by Proposition 4.3, we have

$$
\begin{aligned}
W^{(2)}(\xi, \eta) & =K^{(2)}(\xi, \eta)+\left\{\mathbf{P}^{(2)}(\xi), \eta\right\}-\left\{\mathbf{P}^{(2)}(\eta), \xi\right\} \\
& =\left(K^{(2)}-\partial \mathbf{P}^{(2)}\right)(\xi, \eta) .
\end{aligned}
$$


If $W^{(2)}$ is to be $\partial^{*}$-closed, then this implies that $\partial^{*}\left(K^{(2)}\right)=\partial^{*} \partial \mathbf{P}^{(2)}$. On the other hand, since $H^{1}\left(\mathfrak{g}_{-}, \mathfrak{g}\right)$ is concentrated in homogeneous degrees less or equal to one and $H^{0}\left(\mathfrak{g}_{-}, \mathfrak{g}\right)=\mathfrak{g}_{-k}$, the Hodge decomposition implies that $\mathbf{P}^{(2)}=\square^{-1} \partial^{*} \partial \mathbf{P}-\partial \alpha_{2}$ for a unique smooth section $\alpha_{2}$ of $\mathscr{A}_{2}$. Moreover, since $\mathrm{P}^{(2)}$ has to have values in $\mathscr{A}_{+}$, it follows that the restriction of $\square^{-1} \partial^{*}\left(K^{(2)}\right)$ to $\mathscr{A}_{-k} \oplus \cdots \oplus \mathscr{A}_{-2}$ must be given by $\partial\left(\alpha_{2}\right)$, which gives a condition on the restriction of $\tau_{i}$ to $T^{i-2} \mathscr{G}_{0}$ for $i \leq 0$. If this is satisfied, then $\alpha_{2}$ is uniquely determined, and we can compute $\mathbf{P}^{(2)}$ as $\square^{-1} \partial^{*}\left(K^{(2)}\right)-\partial \alpha_{2}$. Let us notice, how simple the latter step gets for $|1|$-graded examples: then there is no $\alpha_{2}$, the entire forms $\mathrm{P}$ and $K_{0}$ are of homogeneous degree two, and so $\mathrm{P}$ is simply obtained in the unique way by the formula $\mathrm{P}=\square^{-1} \partial^{*} K_{0}$ promised in Example 4.7.

Now this process can be easily iterated. We next consider $K^{(3)}$ which depends only on the (known) component $\mathbf{P}^{(2)}$ of the Rho-tensor and on the restrictions of $\tau_{i}$ to $T^{i-3} \mathscr{G}_{0}$ for $i \leq 0$. As above, the restriction of $\square^{-1} \partial^{*}\left(K^{(3)}\right)$ to $\mathscr{A}_{-k} \oplus \cdots \oplus \mathscr{A}_{-3}$ must be given by $\partial\left(\alpha_{3}\right)$ for a section $\alpha_{3}$ of $\mathscr{A}_{3}$, which gives conditions on the restrictions of $\tau_{i}$ for $i \leq 0$. If these are satisfied, $\alpha_{3}$ is uniquely determined, and we can compute $\mathrm{P}^{(3)}$. Finally, once we have reached $K^{(k)}$, there are no more conditions, since $\tau_{\leq 0}$ is already completely determined at this stage, so we only get a way to compute the remaining homogeneous components of the Rho-tensor.

\section{REFERENCES}

1. Bailey, T. N., Eastwood, M. G., Complexparaconformal manifolds: their differential geometry and twistor theory, Forum Math. 3 (1991), 61-103.

2. Bailey, T. N., Eastwood, M. G., Gover, A. R., Thomas's structure bundle for conformal, projective and related structures, Rocky Mountain J. 24 (1994), 1191-1217.

3. Baston, R. J., Almost Hermitian symmetric manifolds, I: Local twistor theory; II: Differential invariants, Duke Math. J. 63 (1991), 81-111, 113-138.

4. Baston, R. J., Eastwood, M. G., The Penrose Transform. Its Interaction with Representation Theory, Oxford Sci. Publ., 1989.

5. Calderbank, D. M. J., Diemer, T., Differential invariants and curved Bernstein-GelfandGelfand sequences, J. Reine Angew. Math. 537 (2001), 67-103.

6. Čap, A., On partially integrable almost $C R$-manifolds of hypersurface type, in preparation.

7. Čap, A., Gover, A. R., Tractor Calculi for parabolic geometries, Trans. Amer. Math. Soc. 354 no. 4 (2002), 1511-1548.

8. Čap, A., Schichl, H., Parabolic Geometries and Canonical Cartan Connections, Hokkaido Math. J. 29 no. 3 (2000), 453-505.

9. Čap, A., Slovák, J., Souček, V., Invariant operators on manifolds with almost Hermitian symmetric structures, I. Invariant differentiation, Acta Math. Univ. Commenianae 66 (1997), 33-69, electronically available at www.emis.de; II. Normal Cartan connections, Acta Math. Univ. Commenianae 66 (1997), 203-220, electronically available at www.emis.de; III. Standard Operators, Differential Geom. Appl. 12 no. 1 (2000), 51-84. 
10. Čap, A., Slovák, J., Souček, V., Bernstein-Gelfand-Gelfand sequences, Ann. of Math. 154 no. 1 (2001), 97-113.

11. Cartan, E., Les espaces à connexion conforme, Ann. Soc. Pol. Math. 2 (1923), 171-202.

12. Chern, S. S., Moser, J., Real hypersurfaces in complex manifolds, Acta Math. 133 (1974), 219-271.

13. Fefferman, C., Parabolic invariant theory in complex analysis, Adv. Math. 31 (1979), 131262.

14. Gauduchon, P., Structures de Weyl-Einstein, espaces de twisteurs et variétés de type $S^{1} \times S^{3}$, J. Reine Angew. Math. 469 (1995), 1-50.

15. Gover, A. R., Invariants and calculus for projective geometries, Math. Ann. 306 (1996), 513-538.

16. Gover, A. R., Invariants and calculus for conformal geometry, Preprint (1998), to appear.

17. Gover, A. R.,Aspects of parabolic invariant theory, in: Proc. Winter School Geometry and Physics, Srni 1998, Rend6. Circ. Mat. Palermo (2) Suppl. 59 (1999), 25-47.

18. Gover, A. R., Graham, C. R., CR calculus and invariant powers of the sub-Laplacian, to appear.

19. Kaneyuki, S., On the subalgebras $\mathfrak{g}_{0}$ and $\mathfrak{g}_{e v}$ of semisimple graded Lie algebras, J. Math. Soc. Japan 45 (1993), 1-19.

20. Kolár, I., Michor, P. W., Slovák, J., Natural Operations in Differential Geometry, Springer 1993.

21. Kobayashi, S., Transformation groups in differential geometry, Springer-Verlag, Berlin, 1972.

22. Kobayashi, S., Nagano, T., On filtered Lie algebras and geometric structures I.; II., J. Math. Mech. 13 (1964), 875-907; J. Math. Mech 14 (1965), 513-521.

23. Kostant, B., Lie algebra cohomology and the generalized Borel-Weil theorem, Ann. of Math. 74 (1961), 329-387.

24. Lee, J. M., Parker, T. H., The Yamabe problem, Bull. Amer. Math. Soc. (N. S.) 17 (1987), 37-91.

25. Morimoto, T., Geometric structures on filtered manifolds, Hokkaido Math. J. 22 (1993), 263-347.

26. Ochiai, T., Geometry associated with semisimple flat homogeneous spaces, Trans. Amer. Math. Soc. 152 (1970), 159-193.

27. Schmalz, G., Slovák, J., The Geometry of Hyperbolic and Elliptic CR-manifolds of codimension two, Asian J. Math. 4 (2000), 565-598.

28. Sharpe, R. W., Differential Geometry, Graduate Texts in Math. 166 (1997).

29. Slovák, J., Parabolic geometries, Research Lecture Notes, Part of DrSc. Dissertation, Preprint IGA 11/97, electronically available at www.maths.adelaide.edu.au, 70pp.

30. Takeuchi, M., Lagrangian contact structures on projective cotangent bundles, Osaka J. Math. 31 (1994), 837-860.

31. Tanaka, N., On the equivalence problem asociated with simple graded Lie algebras, Hokkaido Math. J. 8 (1979), 23-84.

32. Thomas, T. Y., On conformal geometry, Proc. Nat. Acad. Sci. U.S.A. 12 (1926), 352-359.

33. Thomas, T. Y., Conformal tensors, Proc. Nat. Acad. Sci. U.S.A. 18 (1931), 103-189.

34. Wünsch, V., On conformally invariant differential operators, Math. Nachr. 129 (1986), 269281 .

35. Yamaguchi, K., Differential systems associated with simple graded Lie algebras, Adv. Stud. Pure Math. 22 (1993), 413-494. 
INSTITUT FÜR MATHEMATIK

UNIVERSITÄT WIEN

STRUDLHOFGASSE 4

A-1090 WIEN

AUSTRIA

DEPARTMENT OF ALGEBRA AND GEOMETRY

MASARYK UNIVERSITY

JANÁČKOVO NÁM. 2A

66295 BRNO

CZECH REPUBLIC

E-mail: slovak@math.muni.cz
INTERNATIONAL ERWINSCHRÖDINGER INSTITUTE FOR MATHEMATICAL PHYSICS

BOLTZMANNGASSE 9

A-1090 WIEN

AUSTRIA

E-mail: Andreas.Cap@esi.ac.at 